\title{
A nonlinear dynamics model for simulating long range correlations of cognitive bistability
}

\author{
Norbert Fürstenau
}

Received: 11 December 2008 / Accepted: 26 March 2010 / Published online: 20 April 2010

(C) The Author(s) 2010. This article is published with open access at Springerlink.com

\begin{abstract}
Simulation results of bistable perception due to ambiguous visual stimuli are presented which are obtained with a behavioral nonlinear dynamics model using perception-attention-memory coupling. This model provides an explanation of recent experimental results of Gao et al. (Cogn Process 7:105-112, 2006a) and it supports their speculation that the fractal character of perceptual dominance time series may be understood in terms of nonlinear and reentrant dynamics of brain processing. Percept reversals are induced by attention fatigue and noise, with an attention bias which balances the relative percept duration. Dynamical coupling of the attention bias to the perception state introduces memory effects leading to significant long range correlations of perceptual duration times as quantified by the Hurst parameter $H>0.5$ (Mandelbrot, The fractal geometry of nature, 1991), in agreement with Gao et al. (Cogn Process 7:105112, 2006a).
\end{abstract}

Keywords Cognitive bistability $\cdot$ Modelling ·

Nonlinear dynamics · Perception - Attention .

Long range correlations $\cdot$ Hurst parameter

\section{Introduction}

\subsection{Bistable perception}

Bistable perception is the spontaneous involuntary switching of conscious awareness between the different percepts of an ambiguous stimulus. It is excited with different methods

\section{N. Fürstenau ( $\square)$}

German Aerospace Center, Institute of Flight Guidance,

Lilienthalplatz 7, 38108 Braunschweig, Germany

e-mail: norbert.fuerstenau@dlr.de

URL: www.dlr.de and stimuli, such as binocular rivalry (Blake and Logothetis 2002), perspective reversal, e.g., with the famous Necker cube (e.g., Orbach et al. 1963; Borsellino et al. 1972; Gao et al. 2006a), and apparent motion displays (Hock et al. 1993, 1997). Tristable perception was investigated by Ito et al. (2003). Investigation of multistability provides a unique approach to fundamental questions of perception and consciousness, e.g., the contributions of bottom-up and top-down processing, because it allows for the direct measurement of the switching of subjective perception under constant external stimulus (e.g., Engel et al. 1999; Srinavasan et al. 1999; Edelman 2004). Some experimental results and theoretical considerations indicate that cognitive multistability is not an irrelevant laboratory curiosity, but that it is a product of functional adaptation and the mechanisms underlying multistable perception might be important also for more general aspects of perceptual dynamics (Richards et al. 1994; Arnold et al. 2007). A better understanding of the thalamocortical and behavioral dynamics of cognitive bistability is also of practical relevance due to its reported occurrence during usage of monocular head-worn displays providing flight information to pilots in advanced augmented vision systems (Patterson et al. 2007).

\subsection{Formal modeling approaches}

Formal mathematical models of multistability usually rely on neural dynamics (Wilson 1999; Deco and Marti 2007), take a neural network perspective (e.g., Natsuki et al. 2000) or use a mesoscopic approach (Schuster and Wagner 1990). The latter authors derived within the framework of a mean field theory a phase shift between the coupled self-oscillating neuronal columns, with phase locking formalized by means of the circle (sin-) map. That is, the system state (e.g., phase difference) at time $t+\mathrm{d} t$ depends nonlinearly (sine 
function) on the state at time $t$. A sine map was also derived by Haken (2002) for pulse coupled neural ensembles. Aspects of the nonlinear system dynamics on the level of neural populations were taken into account by several authors (Hock et al. 2003; Noest et al. 2007; Deco and Marti 2007). The latter authors formalized the interaction between two populations of neurons by Wilson-Cowen type first-order nonlinear stochastic differential equations including additive Gaussian noise, however without explicitly regarding attention fatigue.

To the best of our knowledge, Ditzinger and Haken (1989) were the first who transferred the dynamics to the macroscopic behavioral level of perception state, attention, and memory control parameters using the so-called slaving principle of synergetics (theory of self-organizing dynamical systems): macroscopic-order parameters are created through the nonlinear interaction of the neural microsystems and, in turn, slave the microscopic behavior into coherent synchronous activity (Haken 1978). Within the framework of synergetics, the dynamics of complex systems are often governed by a few macroscopic-order parameters only. The order parameter principle leads to the picture of perception states moving as particles within an energy potential landscape, with energy minima determining stationary states. The synergetic-order parameter approach was used recently by Frank et al. (2008) for modeling differential learning within the framework of self-organizing dynamical systems. Within this physical framework of cognitive dynamics, Einstein's fluctuation-dissipation theorem prescribes noise to play a central role in the dynamical evolution via coupling of the dissipative (energy loss) parameters with the noise power (Haken 1978). The importance of noise has been recognized also in recent experiments (Braskamp et al. 2006) and modeling approaches of Noest et al. (2007). In the present approach, it is taken care of within a nonlinear Langevin force differential-delay equation for the attention control parameter. Another possibility would be a Fokker-Planck equation for the time evolution of the probability distribution of the attention parameter (Haken 1978).

Complimentary to the mechanical particle metaphor physiologically based continuum models (Robinson 2005) arrive at macroscopic neural excitation fields, such as formalized by the earlier Jirza-Haken-Nunez (JHN) equation (Jirsa and Haken 1996, 1997) based on pulse coupling within the neural nets (Haken 2002), and on corresponding wave equations of the dendritic current fields. Haken (2002) demonstrated that the field equations can be used to bridge the gap between the neuronal level and phenomena at the behavioral level. The continuum approach yields explanations for a number of EEG characteristics of the modular thalamocortical system (Robinson 2005) and it provides a possible explanation for binocular rivalry via soliton (particle like) dynamics within visual area V1 (Loxley and Robinson 2009).
During the recent years, an increasing number of experimental and theoretical results indicate the chaotic and fractal character of brain functioning (Richards et al. 1994; Lutzenberger et al. 1995; Dafilis et al. 2001; Burke and de Paor 2004; Freeman 2000; Gao et al. 2006a). This appears not surprising, considering the $10^{11}$ neurons with nonlinear transfer characteristic constituting the brain hardware, each one coupled with $10^{3}-10^{4}$ others so that the dynamics of a massive nonlinear delayed feedback system has to be taken into consideration.

\subsection{The behavioral dynamics picture}

Recently, Gao et al. (2006a) presented experimental evidence for the fractal and chaotic character of brain dynamics. It was obtained from analysis of perceptual dwell time series of ambiguous stimuli, such as the Necker cube and binocular rivalry via determination of the self-similarity (Hurst) parameter H (Mandelbrot 1991; Beran 1992). They concluded that nonlinear brain dynamics can be assumed as origin of the corresponding long range correlations.

The present theoretical work supports this conclusion. It predicts long range correlations, i.e., the fractal character of the perceptual duration time series using nonlinear time series analysis applied to results of computer experiments with the formal perception-attention-memory (PAM) model described in Sect.2. Following ideas proposed by von der Malsburg (1997) and Engel et al. (2001), and motivated by the above-mentioned field theoretical approaches (Jirsa and Haken 1996, 1997) in the present model perception states $P_{i}, i=1,2$, are assumed to arise from superimposed coherent fields of synchronously firing neuronal assemblies as excited by the ambiguous stimulus.

Like in Ditzinger and Haken (1989), the basic model couples the dynamics of a macroscopic (behavioral) perception-state-order parameter with an adaptive attention (feedback gain) control parameter with additive noise (Fürstenau 2003, 2004). The order parameter dynamics is derived from a radically simplified field equation with phase feedback of superimposed fields which formalize the potential percepts. The perception state-order parameter is represented by the dynamical phase difference between the two superimposed waves with sufficient degree of coherence. Superimposition yields a recursive nonlinear mapping function which is similar to the phase oscillator equation (sine or circle map) describing the coupling of two (groups of) periodically spiking neurons (Haken 2002) and to coupled nonlinear oscillators, in general (Anishchenko et al. 2003). A similar mapping function which is closely related to the perception state equation of our model was derived by Haken et al. (1985; HKB or phase attractive circle map), and deGuzman and Kelso (1991). It describes the order parameter dynamics of a special 
sensory motor feedback loop. For an overview with further references, see Haken (2002) and Kelso et al. (1995).

The mapping function also shows some similarity to the perception state equation of Atmanspacher et al. (2004) which was derived by means of a so-called weak quantum mechanical approach. In particular, three different time scales of 30-40 ms (feedback delay), 150-200 ms (onset of conscious perception), and $1-3 \mathrm{~s}$ (percept duration) of the present model are in agreement with those of the Atmanspacher model. However, in contrast to Atmanspacher et al. (2004) and in agreement with Ditzinger and Haken (1989, 1995), the present model utilizes explicit perception-attention coupling. Like in Ditzinger and Haken $(1989,1995)$, a perception bias balances the preference between alternative percepts, which in turn is adaptively coupled with the perception state for generating a memory and learning function. The adaptive bias is the origin of the observed long range correlations, leading to self-similar fractal dwell time series.

Multiplicative stimulus strength and adaptive feedback gain (attention parameter) also contrast with the dynamical model of Hock et al. (2003). These authors use an additive stimulus and nonlinearity only present in the coupling between the alternative percepts. Within the present model, the experimentally observed difference of the perceptual duration time statistics between binocular rivalry and perception reversal with regard to the stochastic and deterministic character (Richards et al. 1994; Gao et al. 2006a) can be explained via different memory time constants of the dynamical attention bias which determines the long range correlations.

\subsection{Time series analysis of computer experiments}

Concerning the interpretation of experimental results of bistable perception, there is an ongoing controversial discussion on the possible deterministic, even chaotic or fractal dynamics of cognitive bistability (Poston and Stewart 1978; Ditzinger and Haken 1989; Wilson 1999; Richards et al. 1994; Fürstenau 2004; Noest et al. 2007) versus dominance of stochastic properties (De Marco et al. 1977; Lehky 1995; Merk and Schnakenberg 2002), as derived from statistical analysis of reversal time series. From the above given overview, it appears reasonable to assume that both deterministic and stochastic contributions to the cognitive system dynamics have to be considered. The Hurst parameter $H$ as used by Gao et al. (2006a) is a convenient parameter to quantify the relative importance of deterministic and stochastic dynamics. For example, $H=0.5$ describes a completely stochastic (random walk) system governed by the laws of Brownian motion without any memory effects, whereas a system with $H=1$ is completely deterministic and the initial conditions completely determine the future development. Difficulties arise from the requirements on the amount and quality of the data to be evaluated by sophisticated methods of nonlinear time series analysis for the quantification of the deterministic content (Gao et al. 2006b,c, 2007).

Many formal models of multistability restrict their comparison with experimental results to the relative frequencies of the perceptual dwell time statistics which usually yields the density of a two-parametric $\Gamma$-distribution as reasonable fitting function. It turns out, however, that quite different approaches (e.g., macroscopic as well as microscopic) yield the $\Gamma$-density, so that this does not appear sufficient for discriminating between different models. That is why the recent results of Gao et al. (2006a) with regard to the self-similar, i.e., fractal characteristics of the dwell time series, i.e., long range correlations or memory effects, appear as a very interesting additional necessary condition for cognitive bistability models. The self-similarity or Hurst parameter $H$ of percept reversal time series can be determined by different methods, and the reliability of the results depends on the underlying dynamical system which often is unknown like in the case of cognitive systems (Gao et al. 2007). That is why the comparison of results obtained by different methods have to be critically discussed. The so-called variance-time method and the evaluation of the $1 / f^{\alpha}$-noise characteristic of the spectral power density as discussed by (Beran 1992) and used by (Gao et al. 2006a) is employed also in this study and compared with a method proposed by Kettani and Gubner (2006). I will discuss the results obtained with these three methods and compare it with the direct evaluation of the autocorrelation function of the numerically determined dwell time series.

In Sect. 2, I describe the theoretical approach, including quantitative estimates of the stationary and oscillatory behavior. Results of computer experiments with simulated perception time series and the statistical analysis of the reversal time series for determining the Hurst Parameter $H$ are presented in Sect. 3, followed by the discussion of the results in Sect. 4 and a conclusion and outlook in Sect. 5 .

\section{Theory}

\subsection{The phase oscillator model}

As a kind of minimum architecture allowing for the emergence of fast quasi-discontinuous state transitions, reentrant perception-attention dynamics with attention fatigue (Orbach et al. 1963; Ditzinger and Haken 1989) and delayed feedback is employed (Fürstenau 2004, 2006), with delay $T$ and feedback gain $g$. In the present model, a macroscopic behavioral approach is used with a dynamical perception-state-order parameter $v$, an adaptive attention control parameter $G \sim g$, and a dynamical perception bias parameter $v_{\mathrm{b}}$ representing short term memory and learning. I will 
motivate this architecture by relating the three dynamical model parameters to thalamocortical reentrant loops as proposed by Tononi and Edelman (1998) and to the bottom-up and top-down aspects of visual attention (Itti and Koch 2001). Figure 1 depicts within a block diagram important modules of the attentionally modulated visual perception system.

The diagram is based on simplified brain circuit schematics (e.g., Robinson 1998) including the attentional top-down modulation of the dorsal ("where") and ventral ("what") bottom-up streams of information. It is assumed that as a first approximation for modeling bistable switching between conscious percepts, feedforward preprocessing of the stimulus up to the Primary Visual Cortex V1 (stimulus-V1 delay ca. $40 \mathrm{~ms}$; Lamme 2003) can be neglected, the relevant processing steps taking place within a recurrent perception-attention loop (150-200 ms total processing time until conscious perception) of the association cortex (e.g., Tononi and Edelman 1998; Itti and Koch 2001; Lamme 2003) and a thalamocortical loop via Hippocampus and limbic system. The latter taken into account memory and learning effects which are assumed to determine the dynamical attention/perception bias or percept preference. For the stationary stimuli under consideration, here, the loop via the superior colliculi (SC) for control of eye movements, i.e., overt attention is neglected. On the other hand, recent experimental results indicate that binocular rivalry involves a more automatic, stimulus-driven form of competition than ambiguous figure reversal, and is less easily biased by selective attention (Meng and Tong 2004). This difference between rivalry and ambiguous figure reversal is experimentally expressed in a different ratio of stochastic versus deterministic character of reversal time series (e.g., Richards et al. 1994). I will demonstrate how this observation can be explained within the present model via the memory time constant $\tau_{\mathrm{M}}$.

Without explicitly considering early feedforward processing, in the present approach, selective attention (i.e., preference of one of the two percepts) may be modeled by the dynamic bias parameter $v_{\mathrm{b}}(t)$ which determines relative percept duration times and long range correlations via the $v_{\mathrm{b}}$-memory time constant $\tau_{\mathrm{M}}$ (see below; Fürstenau 2007). Because the model architecture is suggested to basically represent the ventral ("what") V4-InferoTemporal (IT)-PraeFrontal (PF)-V4 loop and the thalamocortical hippocampal (memory) loop as representative target structures it consequently favors a top-down view assuming the usage of prior knowledge (memory) according to Hamker (2004). For simplicity in its present form, it neglects possible early spatial attention modulation of perception reversals, although the latter effect is indicated by recent neurophysiological measurements (Pitts et al. 2007). The model also neglects eye movements and saccades which possibly influence the perception dynamics, e.g., via the thalamocortical loop PF-SCEye-LGN, and via basal ganglia back to the cortex. On the other hand, it has the potential for explaining microsaccadic and tremor oscillations as a top-down effect through the $\mathrm{SC}$ via thalamocortical limit cycle oscillations (see Sect. 2.2, Fig. 5 and Sect. 3.1, Fig. 6).

Experimental evidence on perception-attention coupling with ambiguous stimuli as assumed here was presented by Nakatani and van Leeuwen (2005) using EEG recording of frontal theta and occipital alpha bands and eye blink rate measurement (Ito et al. 2003). Furthermore, according to Hillyard et al. (1999), stimulus-evoked neuronal activity can be modified by an attentionally induced additive bias or by a true gain modulation (present model parameters $v_{\mathrm{b}}(v)$ and $\left.G\left(v, v_{\mathrm{b}}\right) \sim g\right)$. Increase of gain $g(t)$ is correlated with increased blood flow through the respective cortical areas. Consequently, in the present model, the feedback gain serves as adaptive control parameter which induces the rapid transitions between the alternative stationary perception states P1 and P2 through attention fatigue (Orbach et al. 1963), which is modeled according to Ditzinger and Haken (1989) via coupling to the perception state $v$.

For deriving the formal behavioral model on the level of macroscopic order and control parameters $v(G)$, a very simple field theoretical approach is used. It is motivated by the successful use of field equations (Jirsa and Haken $1996,1997)$ for deriving a correspondence to the phenomenological-order parameter equation employed for modeling a movement control experiment (periodic finger response to acoustic stimuli) of Kelso et al. (1992). The present simplification is based on the hypothesis that the relative phase of superimposed electric fields of sufficient degree of coherence captures the relevant dynamics of perception-state-order parameter(s). Consequently, as a first approximation, the superimposition of plane waves as idealized representations of possible perception states is assumed to yield the actual percept after recurrent processing between the thalamocortical modules of Fig. 1 with feedback gain $g \sim G$ and delay time $T$.

An ambiguous stimulus with strength $I \sim G$ and difference-of-meaning $\mu$ of the two possible percepts P1, P2 as stimulus parameter $(0 \leq \mu \leq 1)$ excites two corresponding fields $a\left(\Phi_{1}\right), b\left(\Phi_{2}\right) \sim \exp \{j(\omega t+\Phi)\}$, with phase difference $\Delta \Phi=\Phi_{1}-\Phi_{2}=\pi v_{t}$. The difference-of-meaning parameter is proportional to contrast $\mu=2 a_{0} b_{0} /\left(a_{0}^{2}+b_{0}^{2}\right)$ of (coherent) electromagnetic fields with field amplitudes $a_{0}, b_{0}$. The degree of coherence depends on the spectral distribution of the fields as detailed in Appendix A1. The nonlinear right-hand side of Eq. 1 (see below) describes the conventional interference $J=|a+b|^{2}$ between two superimposed coherent fields (see Appendix A1).

A recurrent process is established by feedback of the output $J$ after amplification (feedback gain $g$ ) with delay $T$ into $\Delta \Phi$ via a hypothetical phase modulation mechanism $\Delta \Phi=\Phi_{\mathrm{t}}+\Phi_{\mathrm{B}}$ and $\Phi_{\mathrm{t}}=\pi J_{\mathrm{t}} / J_{\pi}=\pi v_{t}$, with $J_{\pi}=$ 


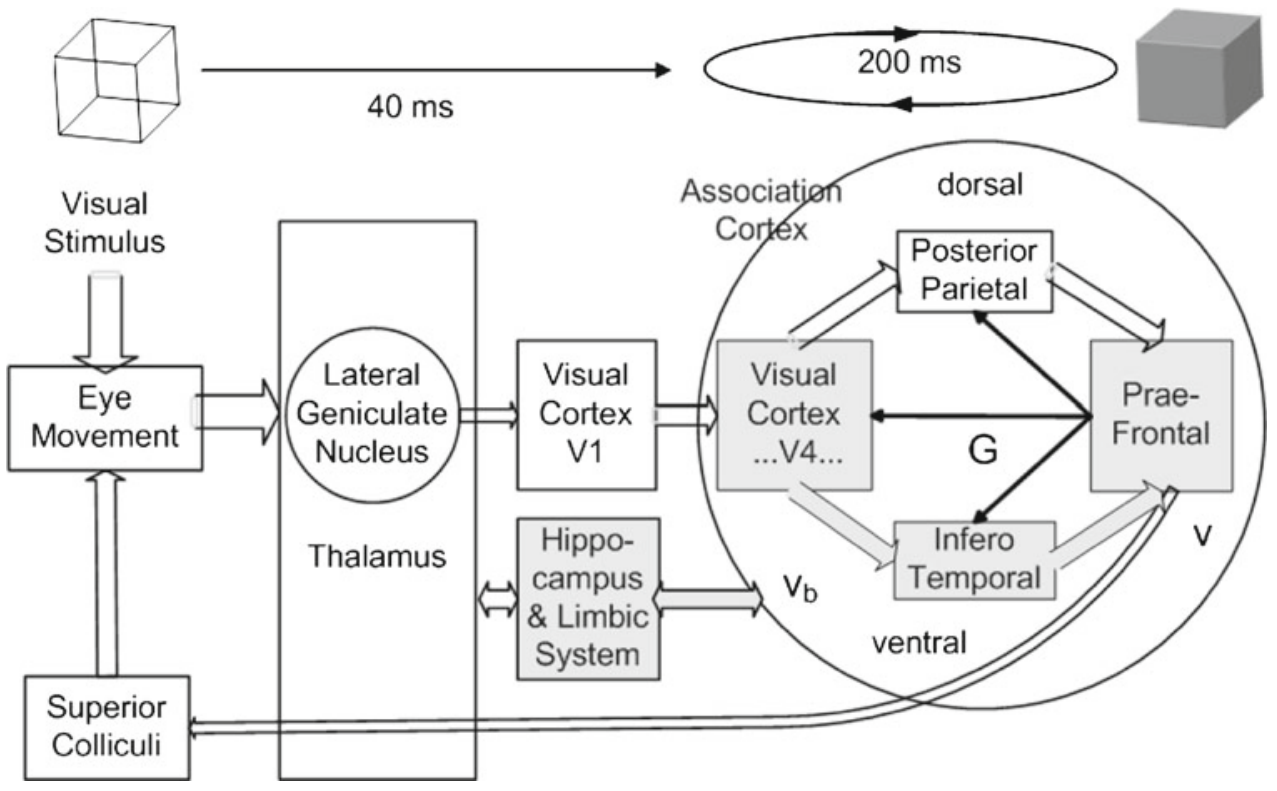

Fig. 1 Schematic representation of essential contributions of visual information flow within the thalamocortical system. Indication of feedforward bottom-up stream $(40 \mathrm{~ms})$ up to $\mathrm{V} 1$ and recurrent processing (150-200 ms) within the association cortex with ventral ("what") and dorsal ("where") pathways including attentional top-down modulation [black arrows; based on Robinson (1998), Itti and Koch (2001), and
Lamme (2003)]. Perception-state order parameter $v$, attention control parameter $G$, and attention bias or preference parameter $v_{\mathrm{b}}$ are placed at neurobiologically relevant positions. Shadowed is main reentrant loop through ventral pathway used as target structure in present model. For details, see text

$\tau_{\mathrm{M}}$ and learning time constant $\tau_{\mathrm{L}}$, respectively, via coupling to the low pass filtered perception state $v$ for allowing adaptive modulation of the bias through the active perception and recognition of initially weakly associated percepts:

$$
\begin{aligned}
& \tau \dot{v}_{\mathrm{t}+\mathrm{T}}+v_{\mathrm{t}+\mathrm{T}}=G\left[1+\mu \cos \left(\pi\left(v_{\mathrm{t}}+v_{\mathrm{B}}\right)\right)\right] \\
& \dot{G}_{\mathrm{t}}=\left(v_{\mathrm{b}}-v_{\mathrm{t}}\right) / \gamma+\left(G_{\text {mean }}-G_{\mathrm{t}}\right) / \tau_{\mathrm{G}}+L_{\mathrm{t}} \\
& \dot{v}_{\mathrm{b}_{\mathrm{t}}}=\left(v_{\mathrm{t}}-\bar{v}_{\mathrm{b}_{\mathrm{t}}}\right) / \tau_{\mathrm{M}}+\left(v_{\mathrm{b}_{\mathrm{e}}}-v_{\mathrm{b}_{\mathrm{t}}}\right) M / \tau_{L}
\end{aligned}
$$

In what follows I assume the phase bias in Eq. $1 v_{\mathrm{B}}=0$ mod 2. The diagram in Fig. 2 represents the highest level (hiding the detailed subprograms) of an implementation of the perception-attention-memory (PAM) equations with the dynamical systems tool Matlab-Simulink.

In agreement with Itti and Koch (2001), the attention parameter $G(v) \sim \kappa I_{0} g(v)$, with detection coefficient $\kappa$, is the product of input (stimulus) strength $I_{0} \quad(=1$ in what follows) and feedback gain $g(v)$. $G$ is coupled with $v$ via fatigue time constant $\gamma$ and recovery time constant $\tau_{G}$. The latter parameter models exponential recovery toward $G_{\text {mean }}=0.5(3-\mu) /\left(1-\mu^{2}\right)=1.875$ for $\mu=0.6$ which is the center between the turning points of the S-shaped stationary hysteresis curve $v^{*}(G)=G\left[1+\mu \cos \left\{\pi\left(v^{*}+v_{\mathrm{B}}\right)\right\}\right]$ (Fürstenau 2004, 2006; see next section, Fig. 3, and Appendix A1). The basic perception-attention dynamics is determined by Eqs. 1 and 2 with fatigue and recovery time constants $\gamma$ and $\tau_{G}$, respectively, feedback delay $T$ and low pass filter a memory and learning function with memory time constant 


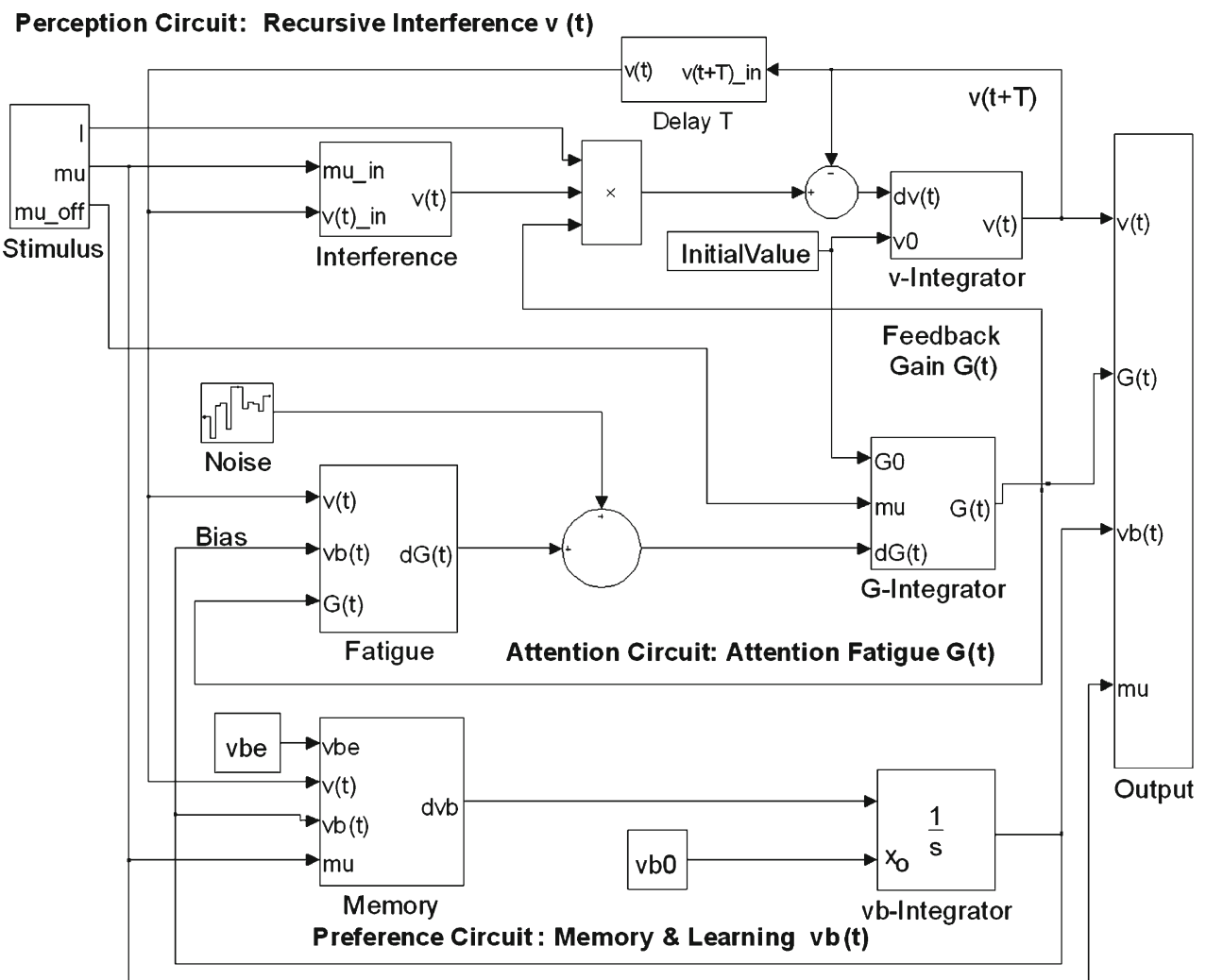

Fig. 2 Simulink implementation of the perception-attention-memory (PAM) Eqs. 1-3. Subroutines (blocks) of the reentrant loops (from top to bottom): perception circuit representing phase oscillator equation $\mathrm{d} v(t) / \mathrm{d} t$, attention circuit representing fatigue equation $\mathrm{d} G(t) / \mathrm{d} t$ with Fatigue and Recovery component, and Preference circuit $\mathrm{d} v_{\mathrm{b}}(\mathrm{t}) / \mathrm{d} t$ representing adaptive bias with Memory and Learning components. Stimulus of strength I and difference-of-meaning mu $(=\mu)$ are fed as control parameters into perception circuit with nonlinear (cosinuidal) interference term and feedback gain $(g \sim G)$ loop with delay $T$ and time constant $\tau$. Attention circuit $G(t)$ with satiation (fatigue) $(v b-v(t)) / \gamma$ and recovery term $\left(G_{\operatorname{man}}-G(t)\right) / \tau_{\mathrm{G}}$ controls as adaptive gain factor the perception dynamics. The Preference (Memory) circuit is coupled with the perception state and modulates attention as a dynamic bias $v_{\mathrm{b}}(t)$ time constant $\tau$. Following Ditzinger and Haken (1989), the random noise due to physically required dissipative processes (Fluctuation-Dissipation Theorem, e.g., Haken 2002) and coupling of the $v-G-v_{\mathrm{b}}$ loops to the rest of the brain is added to the attention equation $G(t)$ as a stochastic Langevin force $L(t)$. Within Matlab-Simulink, it is realized by the subprogram (block) "Noise" (Fig. 2) with band limited white noise power $J_{\omega}$ and sampling time $t_{\mathrm{c}}$ ( covariance $s^{2}=J_{\omega} / t_{c}$ ).

The dynamic attention bias $v_{\mathrm{b}}(v)$ determines the relative preference of $\mathrm{P} 1$ and $\mathrm{P} 2$. The dynamics $\mathrm{d} v_{\mathrm{b}} / \mathrm{d} t$ is modeled as the sum of a learning function $M\left(v_{\mathrm{t}}, v_{\mathrm{b}}, v_{\mathrm{be}}\right)\left(v_{\mathrm{be}}-v_{\mathrm{b}}\right) / \tau_{L}$, and a short term memory component $\left.\left(<v_{t}\right\rangle-v_{\mathrm{b}}\right) / \tau_{\mathrm{M}}$ which couples $v_{\mathrm{b}}$ to the perception state $v(<>=$ low pass filter). Learning is active only $\left(M\left(v_{\mathrm{t}}, v_{\mathrm{b}}, v_{\mathrm{be}}\right)=0 \rightarrow 1\right)$ if one of the two percepts dominates whereas the other is initially weakly associated (initial bias $v_{\mathrm{b} 0} \neq v_{\text {be }}$, with $v_{\text {be }}=$ equilibrium bias, and $\left.\left|\left\langle v_{\mathrm{t}}\right\rangle-v_{\text {be }}\right|>\left|<v_{\mathrm{t}}\right\rangle-v_{\mathrm{bt}} \mid\right)$, and a fluctuation-induced jump (due to stochastic noise and high frequency limit cycle and chaotic oscillations, see Figs. 5 and 6) into the weak perception state occurs, switching $M$ from 0 to 1 for the duration of the weak state until $\mid<v_{\mathrm{bt}}>-$ $v^{*}(\mathrm{P} 1)|\approx|<v_{\mathrm{bt}}>-v^{*}(\mathrm{P} 2) \mid$ at which time $M$ is switched back: $M=1 \rightarrow M=0$. This feature will gradually move the dynamic bias $v_{\mathrm{b}}(t)$ into the more symmetric equilibrium position $v_{\text {be }} \approx\left(v^{*}(\mathrm{P} 1)+v^{*}(\mathrm{P} 2)\right) / 2$ (see Sect. 3, Fig. 7).

Although it has been shown in previous publications (Fürstenau 2006, 2007) that this minimum version of the model correctly predicts a number of experimental results (e.g., $\Gamma$-distribution of percept dwell times), it clearly has certain limitations, such as the asymmetry of the pitchfork bifurcations on the P1 and P2 levels of the $v^{*}(G)$-topology (see Fig. 3b below) which results in different oscillation amplitudes superimposed to the stationary P1, P2 states. Consequences of the present simplification and a method to overcome the limitations will be discussed in Sect.4. Here, I will follow this parsimonious approach making use of the reduced number of parameters with correspondingly reduced complexity and simulation time. 

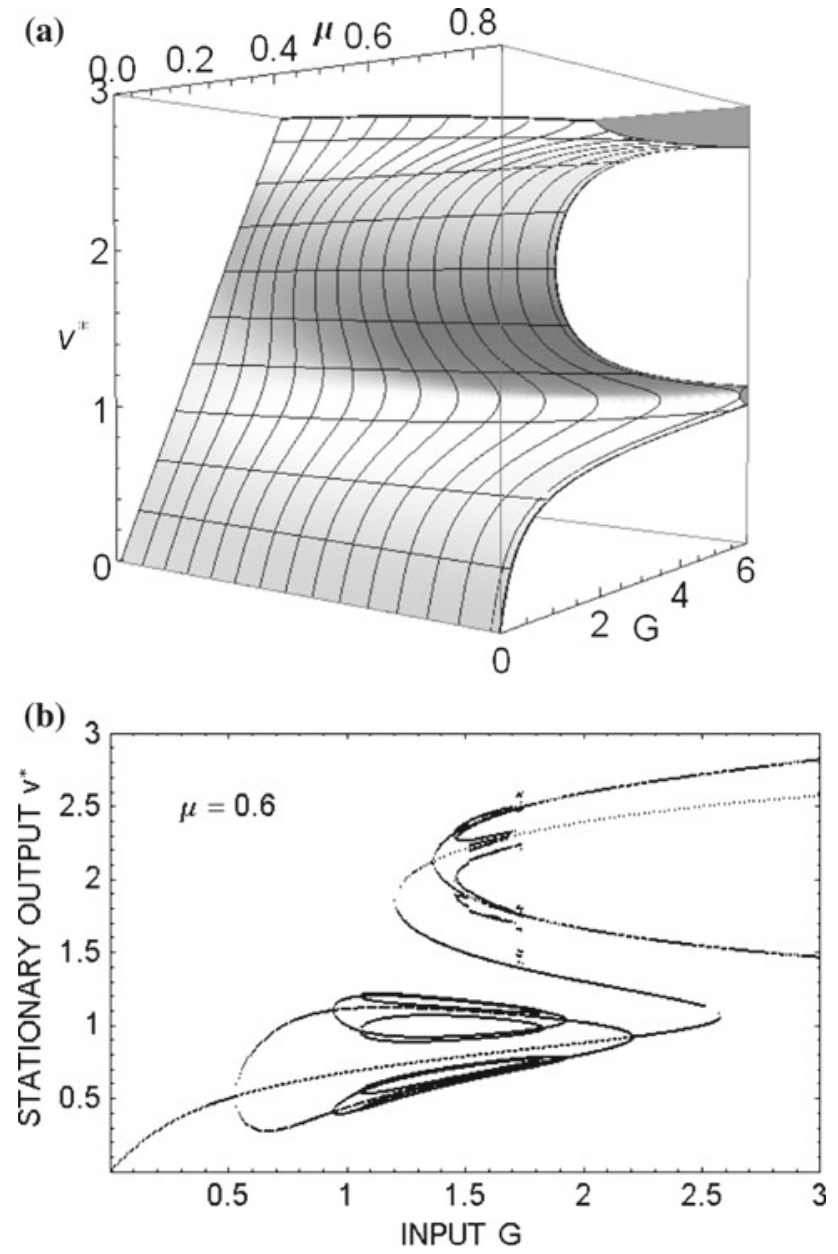

Fig. 3 a Stationary solution $v^{*}$ of perception state equation (1) in the form of a phase parametric diagram as dependent on attention control parameter (feedback gain) $G$ and difference-of-meaning (interference contrast) $\mu$ of percepts $\mathrm{P} 1, \mathrm{P} 2$. Node bifurcation at $\mu_{\mathrm{n}} \approx 0.18$ defines transition from unambiguous to ambiguous solution $((\mu<$ $\left.\mu_{\mathrm{n}}\right)=$ stimulus-off $\rightarrow\left(\mu>\mu_{\mathrm{n}}\right)=$ stimulus-on $)$ with positive slope regions $v^{*}(G)$ representing stationary perception states P1 (lower level $v^{*} \approx 1$ ), P2 (higher level $v^{*} \approx 2.5-3$ ). Dark gray: instable regions. b 2D-cross section through stationary 3D-plot a at stimulus parameter position $\mu=0.6$, exhibiting hysteresis (with $v^{*}(G)$ ambiguity between $G=1.2$ and $G=2.6$ ) as first-order stationary solution and higher order periodical stationary solutions (period doubling bifurcations) $v^{i *}, i=1,2,4,8$

\subsection{Stationary solutions, bifurcations, and oscillations}

In what follows I will first discuss the stationary solution of the perception state equation (1) (the derivation is given in Appendix A1). It will be followed by an analysis of two kinds of oscillations observed in the simulations: (a) bifurcations into low amplitude high frequency limit cycle, period doubling, and chaotic oscillations superimposed on the stationary solutions, with periods of the order $T$; (b) low frequency self-oscillations (percept switching) between the stationary perception states $\mathrm{P} 1, \mathrm{P} 2$ due to attention fatigue $G(t)$ with time constant $\gamma$. The time series analysis of numerical simulations in Sect. 3 will reveal that the limit cycle and chaotic oscillations exhibit only weak influence on the percept dwell time statistics. It will be shown that the latter is determined primarily by the time constant $\gamma$ and the dynamic bias $v_{\mathrm{b}}(t)$ of Eq. 3 together with the additive attention noise $L_{\mathrm{t}}$ (Langevin force) of Eq. 2 with power spectral density $J_{\omega}$.

Low frequency quasiperiodic switching between two stationary perception states $v^{*}(\mathrm{P} 1)$ and $v^{*}(\mathrm{P} 2)$ (self-oscillating percept reversals) of some seconds dwell time emerges after a node bifurcation of the stationary $v^{*}(G, \mu)$ graph as depicted in Fig. 3a, at the stimulus value (bifurcation parameter) $\mu=\mu_{n} \approx 0.18 . v^{*}(G)$ evolves from a monotonous function (for $\mu<\mu_{\mathrm{n}}$ ) into a hysteresis S-shaped ambiguous one with increasing $\mu$ (Fürstenau 2003, 2004, 2006). Figure 3 a depicts the first-order stationary solutions $v^{*}(G, \mu)$ (obtained by setting $\mathrm{d} v / \mathrm{d} t=0, v_{\mathrm{t}+\mathrm{T}}=v_{\mathrm{t}}=v^{*}$, see Appen$\operatorname{dix} \mathrm{A} 1)$.

At $\mu=\mu_{\mathrm{n}} \approx 0.18$, the slope of the stationary system state $\mathrm{d} v^{*} / \mathrm{d} G$ becomes infinite with $\left(G_{\mathrm{n}}, v_{\mathrm{n}}\right) \approx(1.5,1.5)$. For $\mu<\mu_{\mathrm{n}}$ both percepts are fused into a single meaning corresponding to an unambiguous stimulus. For $\mu>\mu_{\mathrm{n}}$, the stationary solution $v^{*}(G)$ becomes multivalued, corresponding to switching-on of the ambiguous stimulus. For maximum contrast $\mu=1$, the horizontal slope $(\mathrm{d} G / \mathrm{d} v)^{-1}=0$ yields $v_{\mathrm{i}}^{\infty}=2 i-1, i=1,2,3, \ldots$ as stationary perception levels in the limit $G \rightarrow \infty$. The node bifurcation and hysteresis agrees with the qualitative deterministic catastrophe theoretical model of cognitive bistability as proposed by Poston and Stewart (1978). It is of interest to note that, like in the model of Noest et al. (2007) with additive stimulus, the present model with multiplicative stimulus parameter $\mu$ exhibits the same qualitative difference between transition into perception states via a choice process (i.e., unambiguous to ambiguous transition, mathematically represented by a node bifurcation $\mu<\mu_{\mathrm{n}} \Rightarrow \mu>\mu_{\mathrm{n}}$ ), and via the fatigue $G(t)$-driven percept reversal in the ambiguous $G$-range with $\mu>\mu_{\mathrm{n}}$ (self-oscillation P1 $\Leftrightarrow \mathrm{P} 2$, see below). In Sect. 3, we will exclusively concentrate on quasiperiodic reversal processes $\mathrm{P} 1 \Leftrightarrow \mathrm{P} 2$, where the periodicity of the self-oscillations are disturbed by the attention noise $L_{\mathrm{t}}$ of the feedback gain (attention parameter) Eq. 2.

Superimposed on the stationary levels $v_{1} *(\mathrm{P} 1), v_{2} *(\mathrm{P} 2)$ is the attention noise $L_{\mathrm{t}}$ as characterized by power spectral density $J_{\omega}$, and additionally the high frequency limit cycle and chaotic oscillations (up to some $10 \mathrm{~Hz}$ ) originating from the feedback delay $T$. The latter contribution is illustrated in Fig. 3b which shows for fixed $\mu=0.6$ higher order stationary solutions $\left(v\left(t+2^{\mathrm{i}} T\right)=v(t)=v^{\mathrm{i}} *, i=\right.$ $0,1,2,3 \ldots)$ exhibiting period doubling pitchfork bifurcations on both positive slope regions of the hysteresis curve. The bifurcation points seem to converge at a chaotic boundary following the same universal law as that one discovered 


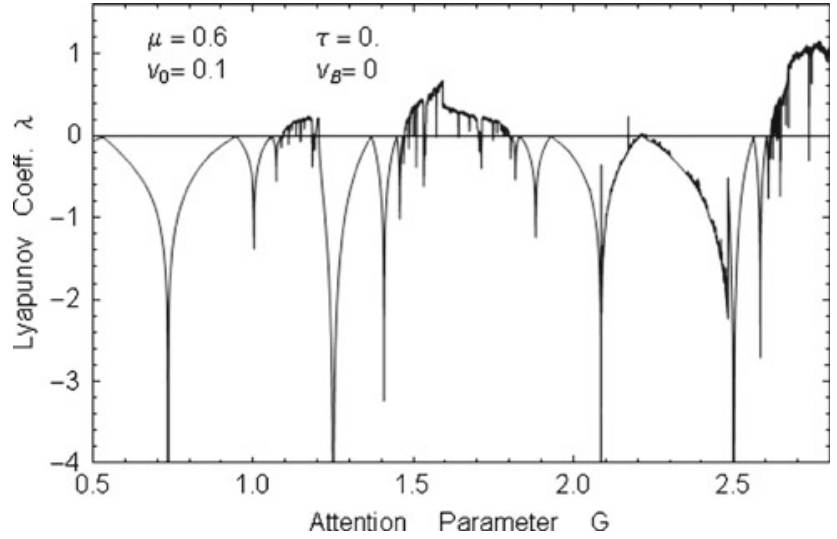

Fig. 4 Plot of Lyapunov exponent according to Eq. (4) with damping time constant $\tau=0$ (discrete map, no damping). Positive $\lambda$ values correspond to chaotic dynamics, observed as superimposed oscillations on stationary levels in Fig. 3b within three control parameter $(G)$ ranges. Stationary level $1: G \approx 1.1-1.2$; level $2: G \approx 1.5-1.8$; level 3 (not shown in Fig. 3): $G>$ 2.6. $\lambda=0$ corresponds to bifurcation points in the stationary solutions of Fig. $3 b$

by Feigenbaum (1979) for the quadratic map (see Appendix A2 for a quantitative evaluation). The bifurcation points $G_{\text {n }}$ of the attention control parameter $G$ and the $G$-ranges of chaotic regimes in the bifurcation graph may be determined either directly from the data of Fig. 3b or by numerical evaluation of the Lyapunov exponent $\lambda$ which is given by (Schuster and Just 2005)

$\lambda\left(v_{0}\right)=\lim _{N \rightarrow \infty} \frac{1}{N} \sum_{i=0}^{\mathrm{N}-1} \ln \left(\left|f^{\prime}\left(v_{\mathrm{i}}\right)\right|\right)$

with $v_{0}=$ start value of iteration, $N=$ number of iteration steps and $f^{\prime}\left(v_{\mathrm{i}}\right)=$ derivative of the right-hand side of the discrete version of Eq. 1: $v_{\mathrm{t}+\mathrm{T}}=f\left(v_{\mathrm{t}}\right)$, where $\mathrm{d} v / \mathrm{d} t$ is replaced by $\left(v_{\mathrm{t}+\mathrm{T}}-v_{\mathrm{t}}\right) / T$ (see Appendix A2). $\lambda$ measures the exponential separation of neighboring initial states $v_{0}$ during the iteration process under the action of the mapping $v_{\mathrm{t}} \rightarrow v_{\mathrm{t}+\mathrm{T}}$. It corresponds to the average loss of information per iteration. Figure 4 shows $\lambda$ for $N=1,000$ iterates with $\tau=0$ as plotted versus $G$. Coming from negative values, $\lambda$ touches the $\lambda=0$ axis at bifurcation points $G_{\mathrm{n}}$ (period doubling) with decreasing distance $G_{\mathrm{n}}-G_{\mathrm{n}-1}$ until it crosses $\lambda=0$ at the boundary to chaotic regimes, characterized by positive $\lambda$.

For finite low pass filter time $\tau>0$, chaotic regions and amplitudes of limit cycle oscillations decrease. This can be quantified by a linear stability analysis of perception equation (1); (e.g., Fürstenau (2003), see also Appendix A3) which yields Eigenfrequencies $\beta=2 \pi f$ via

$\beta \tau=-\tan (\beta T)$.

The numerical solution for $\tau=1 T_{\mathrm{S}}, T=2 T_{\mathrm{S}}$, with simulation time steps $T_{\mathrm{S}}=T / 2=20 \mathrm{~ms}$ (Fürstenau 2006, 2007) yields for the lowest frequencies $f / H z=9.1,20.2,32.2$, and 44.5. For $i<10$, and dependent on $\tau / T$, the spectrum lies well within the range of typical EEG frequency bands as well as those of microsaccades $(3-4 \mathrm{~Hz})$ and tremor $(30$ $150 \mathrm{~Hz}$ ) of fixational eye movements. This demonstrates that also the oscillatory characteristics of the stationary states possibly reflects observable physiological brain activities as well as eye movement. The computer experiments (simulations) in Sect. 3 seem to show the expected chaotic behavior on the higher stationary level (P2-state) as depicted in the inset of Fig. 6a, whereas on the lower (P1) level the predicted limit cycle oscillations around $10 \mathrm{~Hz}$ dominate. This difference can be explained by the relatively high low pass filter time constant $\tau / T>0.1$ [see Appendix A3 and Fürstenau (2003)].

The limit cycle and chaotic characteristics of the fast perception state oscillations as revealed in the plot of the Lyapunov exponent (Fig. 4) is supported by the $v(t+T)$ versus $v(t)$ phase space plots of Fig. 5a and $\mathrm{b}$ for one period of percept reversal. They are obtained by numerical solutions of the PAM equations (1)-(3) for two different values of the time constants $\tau$ and $\gamma$ (in units $T_{\mathrm{S}}=20 \mathrm{~ms}$ ) and with zero noise $\left(L_{\mathrm{t}}=0\right)$.

Both figures clearly exhibit separated phase space regions for the P1 (bottom left: $v(t), v(t+T)<\approx 1$ ) and P2 $(v(t), v(t+T)>\approx 1)$ states. It can be seen that even without noise $\left(L_{\mathrm{t}}=0\right)$, the areas are increasingly filled by the trajectories with decreasing damping time constant (Fig. 5a: $\tau=1$, Fig. 5b: $\tau=0.1$ ) and with increasing fatigue time constant $\gamma$ (increasing percept dwell time) which determines the percept trajectory length in phase space per dwell time period. Besides the transition to a positive valued Lyapunov exponent as depicted in Fig. 4, the filling of the phase space (ergodic trajectories) is another indication of chaotic behavior (Schuster and Just 2005). The chaotic behavior of idealized technical systems with time-delayed feedback and zero noise as described by our perception state equation (1) was investigated in detail both theoretically (Ikeda and Matsumoto 1987) and experimentally with electro-optical devices (Derstine et al. 1987), so that we refer to these publications for further details. An advanced method for distinguishing chaos from noise by the scale-dependent Lyapunov exponent which is suitable also for short time series was presented by Gao et al. (2006c). In our case, it would be useful, e.g., for analysis of stationary perception states with noise term $L_{\mathrm{t}} \neq 0$ which might become relevant if we include in the simulation feedforward processing and the loop via SC and LGN for modeling (fixational) eye movement (see Fig. 1). This is, however, neglected within the present context as mentioned before.

An estimate of the expected low frequency perceptual selfoscillations between the stationary states $v^{*}(\mathrm{P} 1) \Leftrightarrow v^{*}(\mathrm{P} 2)$ due to the $v-G$ coupling and attention fatigue $G(v)$ with time constant $\gamma=1.2 \mathrm{~s}$ may be obtained by combination 

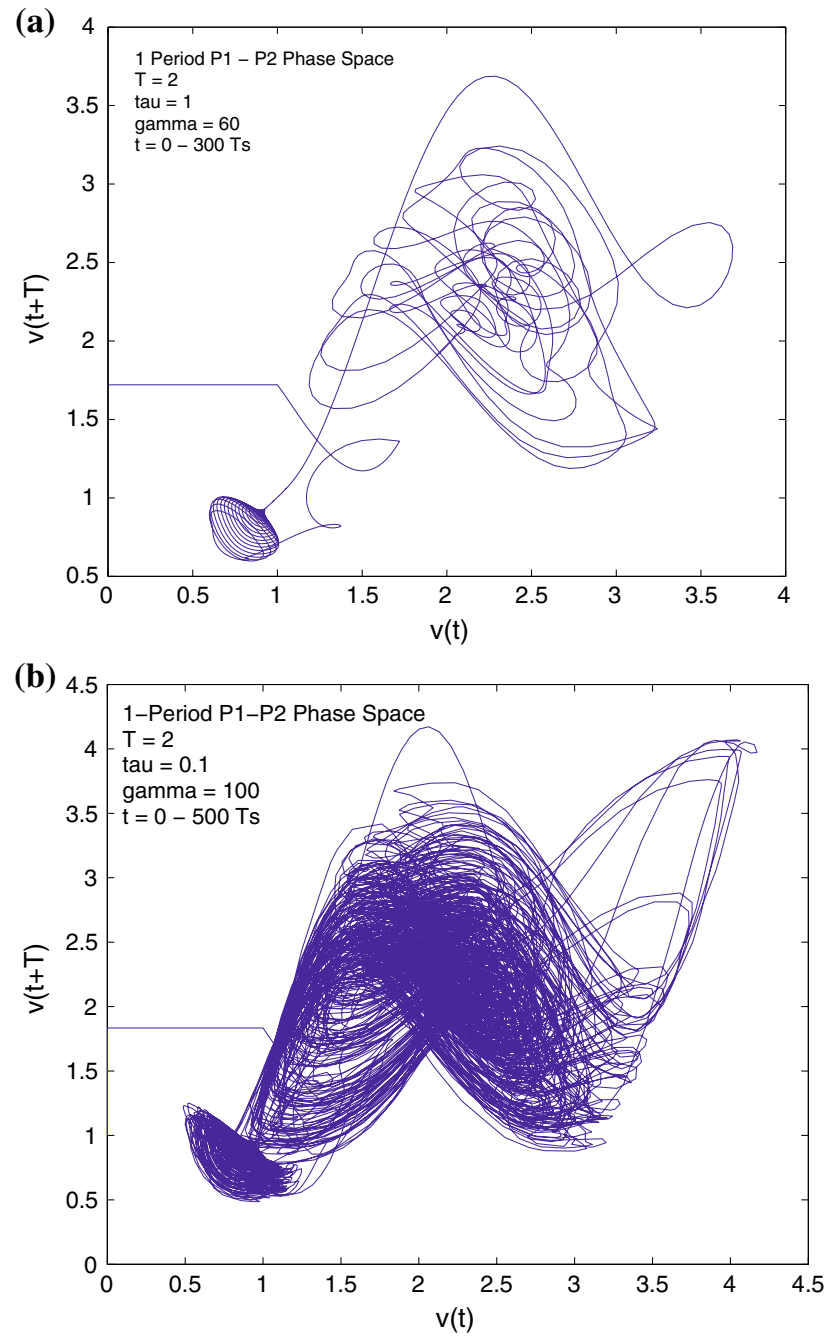

Fig. 5 Phase space plot of perception states $\mathrm{P} 1$ (lower left area, $v_{\mathrm{t}}<\approx$ 1 ), $\mathrm{P} 2$ (upper right region, $v_{\mathrm{t}}>\approx 1$ ) with zero noise for one $\mathrm{P} 1-\mathrm{P} 2$ period. a Time series $t=0-300 T_{s}=6 \mathrm{~s}$, with large damping time constant $\tau=1$, fatigue $\gamma=60$, exhibiting damped limit cycle oscillations of P1 and filling up of P2 phase space area. b Time series $t=$ $0-500 T_{s}=10 \mathrm{~s}$, with decreased damping time $\tau=0.1$, increased fatigue time constant $\gamma=100$ (i.e., increased dwell time)

of Eqs. 1 and 2 and derivation of the eigenfrequency of the linearized differential-delay equation (see Appendix A4):

$f_{\mathrm{D}}=\frac{1}{2 \pi} \sqrt{\frac{1-D^{2}}{\gamma(\tau+T)}}=f_{0} \sqrt{1-D^{2}}$

with $f_{0}=0.59 \mathrm{~Hz}=36 \mathrm{~min}^{-1}$ or period $T_{0}=1.7 \mathrm{~s}$ and normalized damping $D=0.358$, yielding the reversal rate $f_{\mathrm{D}}=0.55 \mathrm{~Hz}=33 \mathrm{~min}^{-1}$. Although, the very rough dwell time estimate for a single percept $\Delta\left(\mathrm{P}_{\mathrm{i}}\right)=T_{\mathrm{D}} / 2=1 / 2 f_{\mathrm{D}}$ due to the low hysteresis $(\mu=0.2)$ lies at the lower end of the typical experimental results it nevertheless predicts the correct order of magnitude (e.g., Orbach et al. 1963; Levelt
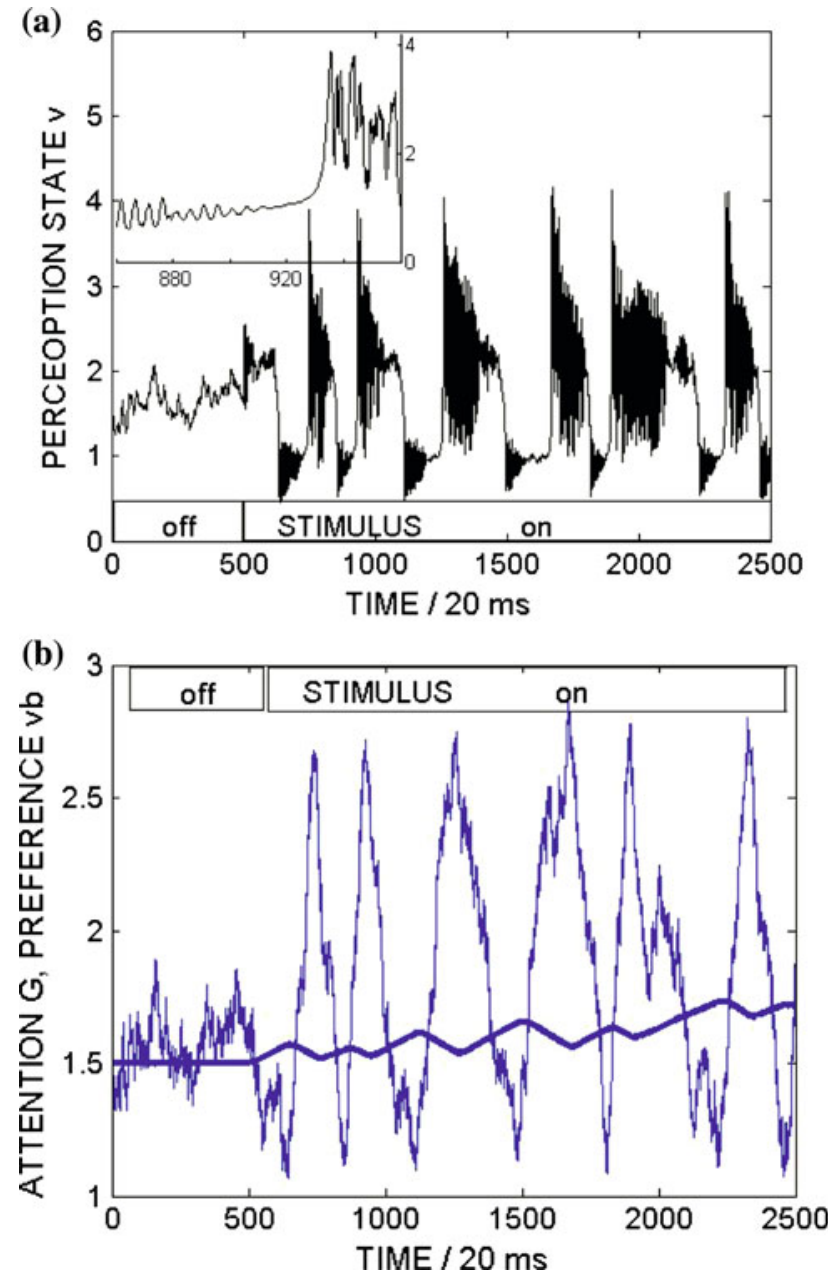

Fig. 6 Numerical evaluation of PAM equations (1)-(3) for time duration $t=2500 T_{\mathrm{S}}=50 \mathrm{~s}\left(T_{\mathrm{S}}=T / 2:=20 \mathrm{~ms}\right)$. See text for simulation parameters. a Perception state $\mathrm{v}(\mathrm{t})$; Stimulus-off $(\mu=0.1)$ during $t=0, \ldots, 500 T_{\mathrm{S}}$, stimulus-on $(\mu=0.6)$ during $t=500-2500 T_{\mathrm{s}}$. Inset shows single transition and oscillations superimposed on stationary levels around $v \approx 1$ and $v=2.5$. b Attention parameter $G(t)$ (Eq. 2) and dynamic attention bias or preference state $v_{\mathrm{b}}$ (thick line) with $v_{\mathrm{b}}$-dynamics (Eq. 3 governed by memory time constant $\tau_{M}$ due to coupling of $v_{\mathrm{b}}$ to $v$ )

1967; Borsellino et al. 1972; De Marco et al. 1977; Zhou et al. 2004).

\section{Computer experiments}

\subsection{Simulated perception-attention-memory dynamics}

In this section, numerical evaluations of the coupled PAM differential-delay equations (1)-(3) are performed with the Matlab-Simulink code of Fig. 2 using the Runge-Kutta solver "ode23tb" for stiff problems, i.e., fast changing dynamics. As an example, Fig. 6a and b shows time series $v(t)$ and $G(t)$ as obtained with the following parameter 
values ( time units $=$ simulation intervall $T_{\mathrm{S}}=T / 2$ ): reentrant delay time $T=2 T_{\mathrm{S}}=40 \mathrm{~ms}$, corresponding to Lamme (2003), stimulus parameter (difference-of-meaning of percepts) $\mu=0.6, \tau=0.5, \gamma=60, \tau_{G}=500$, equilibrium perception bias $v_{\text {be }}=1.5$, Langevin force $L_{\mathrm{t}}$ with noise power $J_{\omega}=0.001$ and sample time $t_{\mathrm{c}}=0.1$ (=noise correlation time), memory time constant $\tau_{\mathrm{M}}=1,000$, learning time constant $\tau_{\mathrm{L}}=100,000$ (i.e., learning negligible). A stimulus-off interval ( $\mu_{\text {off }}=0.1, G_{\text {off }}=1.5$ ) is selected at the beginning of the time series, with initial bias $v_{\mathrm{b} 0}=v_{\mathrm{b}}(t=0)=1.5$.

After stimulus-on at simulation time $500 T_{\mathrm{S}}$, the $v(t)$ dynamics in Fig. 6a immediately starts the quasiperiodic transitions between stationary perception states $\mathrm{P} 1$ (near $v^{*} \approx 1$ ) and $\mathrm{P} 2$ (near $v^{*} \approx 2.5$ ) with the expected superimposed mixture of fast limit cycle and chaotic oscillations and noise. The inset in Fig. 6a shows that the transition time between P1 and $\mathrm{P} 2$ is of the order of $8-10 T_{\mathrm{S}} \approx 150-200 \mathrm{~ms}$, in reasonable agreement with the time interval between stimulus onset and conscious perception (Lamme 2003). The superimposed fast oscillations exhibit a damped characteristic and the dominating component on the $\mathrm{P} 1$ state can be seen to agree with the ca. $10 \mathrm{~Hz}$ estimate (dominating Eigenfrequency of Eq. 5) obtained from the linear stability analysis.

For determining percept dwell time statistics and long range correlations in Sect. 3.2100 time series like in Fig. 6a (however without stimulus-off phase) with simulation length of $5000 T_{\mathrm{S}}(=100 \mathrm{~s})$ and each with different noise seed and initial values $v_{0}=v(t=0), G(t=0)=G\left(v_{0}^{*}\right)\left(v_{0}=\right.$ $1.5000 \ldots 1.5099)$, were evaluated per fixed set of parameters after concatenation by setting bias (memory) parameter $v_{\mathrm{b}}\left(t=0 T_{\mathrm{S}}\right)$ of time series $n+1$ equal to $v_{\mathrm{b}}\left(t=5000 T_{\mathrm{S}}\right)$ of time series $n(n=1, \ldots, 100)$. In this way, effective simulation length per fixed parameter run corresponded to ca. $160 \mathrm{~min}$, yielding typically 1,300 perception intervals P1 and P2. The limitation of time series length was selected due to computer memory requirements under Matlab version 5.2 and for avoidance of excessive computation time (computer hardware: Pentium (R) Dual Core, 3.2 GHz, 2 GB RAM, Windows XP).

The attention control parameter $G(t)$ ( adaptive feedback gain, sawtooth graph in Fig. 6b) exhibits the slow fatigue dynamics which induces the fast quasiperiodic P1 $\Leftrightarrow \mathrm{P} 2$ transitions at the G-extrema according to the stationary hysteresis curve of Fig. 3a and 3b. The relative P1/P2-duration is modulated by the adaptive attention bias $v_{\mathrm{b}}(t)$ (thick line, starting at $\left.v_{\mathrm{b} 0}=1.5\right)$. $v_{\mathrm{b}}$ induces a memory effect due to coupling to the low-pass filtered perception state $\langle v(t)>$. The mean P1-P2 reversal frequency is determined by stimulus-on contrast parameter $\mu=0.6$ (difference-of-meaning), the fatigue time constant $\gamma(=1 / \mathrm{c}$ in Ditzinger and Haken (1989)), damping time $\tau$, and delay $T$. A typical full period of $\Delta=\Delta(\mathrm{P} 1)+\Delta(\mathrm{P} 2) \approx 300 T_{\mathrm{S}}=6 \mathrm{~s}$ is observed in

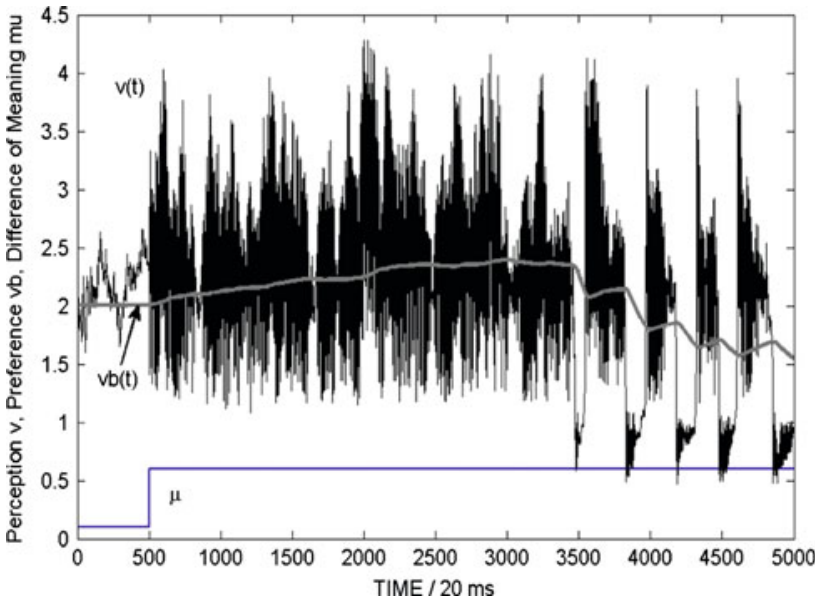

Fig. 7 Simulation with strong initial bias toward percept P2: $v_{\mathrm{b} 0}=$ 2.0, $v(t=0)=2.0$. Learning dynamics activated by selecting small learning time constant $\tau_{\mathrm{L}}=400 T_{\mathrm{S}}$. Figure shows three time series $v(t), v_{\mathrm{b}}(t), \mu(t)$. Fluctuations induce jumps from P2 into P1 starting at $t \approx 3500 T_{\mathrm{S}}$ (initial perception of $\mathrm{P} 1$ ) gradually force adaptive bias (memory) parameter $v_{\mathrm{b}}$ to the equilibrium value (preprogrammed memory value) $v_{\text {be }}=1.5$ with onset of percept reversals due to coupling of $v_{\mathrm{b}}$ to $v$ via $\tau_{\mathrm{L}}$ and additional memory effect with time constant $\tau_{\mathrm{M}}$

Fig. 6 which corresponds to the typical experimental observations reported in the literature. It also agrees with the order of magnitude of the linearized analytical estimate (Eq. 6) which for small $\mu(=0.2)$ and zero noise (Eq. 2: $L_{\mathrm{t}}=0$ ) yields $\Delta=1.8 \mathrm{~s}$ in reasonable agreement with the corresponding simulation result of $\Delta(\mathrm{P} 1+\mathrm{P} 2)=1.3 \mathrm{~s}$ for zero noise and $\mu=0.23$, i.e., nearly vanishing $v^{*}-G^{*}$ hysteresis (see Fig. 3a). The significant influence of noise in agreement with Braskamp et al. (2006) is demonstrated through the fact that the simulation with small stimulus parameter (hysteresis) $\mu=0.2$ and noise $L_{\mathrm{t}} \neq 0$ (power $J_{\omega}=0.004$, sampling time $\left.t_{\mathrm{c}}=0.1 T_{\mathrm{S}}\right)$ yields the full period $\Delta(\mathrm{P} 1+\mathrm{P} 2) \approx 2.8 \mathrm{~s}$, still half the value obtained with $\mu=0.6$, but doubled as compared to the simulation without noise $\left(J_{\omega}=0\right)$.

The fatigue dynamics $\mathrm{d} G / \mathrm{d} t$ due to coupling to $\left(v_{\mathrm{b}}-v\right)$ with time constant $\gamma$ is inhibited by the exponential-recovery process $\left(G_{\text {mean }}-G\right)$ with time constant $\tau_{G}$. With the time constant values used in the present simulations $\left(\gamma / \tau_{\mathrm{G}} \approx 10\right)$, the inhibition of fatigue due to the recovery is small.

As a second example for the numerical solution of the PAM equations, Fig. 7 shows a simulation with strong bias toward percept P2 by selecting initial value $v_{\mathrm{b} 0}=2$ and activating learning dynamics by selecting a small learning time constant $\tau_{\mathrm{L}}=400 T_{\mathrm{S}}$. Three superimposed curves are shown representing the perception state $v(t)$ and perception bias $v_{\mathrm{b}}(t)$, together with the contrast function $\mu$ (stimulus parameter: difference-of-meaning) which switches from $\mu=$ 0.1 (stimulus-off) to $\mu=0.6$ (stimulus-on) at $500 T_{\mathrm{S}}$. The curves show how the initial P2-state with high frequency oscillations around the first-order stationary $v$-level near $v=$ 
2.5 (corresponding to a situation with an initially unknown P1-state) eventually starts low frequency $v(t)$-self-oscillations $\mathrm{P} 2 \Leftrightarrow \mathrm{P} 1$ at time $t \approx 3500 T_{\mathrm{S}}=70 \mathrm{~s}$ under gradual change of $v_{\mathrm{b}}$ toward the equilibrium state $v_{\mathrm{be}}=1.5$. This is due to the indirect coupling of $v(t)$ to the perception bias $v_{\mathrm{b}}(t)$ via attention control parameter $G(t)$. The onset of selfoscillations ( $\mathrm{P} 2 \Leftrightarrow \mathrm{P} 1)$ with significant $v_{\mathrm{b}}$-deviation from the equilibrium value $v_{\text {be }} \approx 1.5$ is possible only due to large $v$-fluctuations now and then originating from the stochastic attention component (Langevin force $L_{\mathrm{t}}$ realized by band limited white attention noise $J_{\omega}$ ). Noise continuously modulates $v_{\mathrm{b}}$ and from time to time together with the chaotic and limit cycle oscillations leads to large enough P2-fluctuations allowing for switches into P1. In the example of Fig. 7, this happens for the first time around $t=3500 T_{\mathrm{S}}$, representing the onset of quasiperiodic self-oscillations. It should be mentioned that the details of the $v(t)$ pattern of the learning phase and $\mathrm{P} 1 \Leftrightarrow \mathrm{P} 2$ self-oscillations can change drastically with the slightest change of the noise seed value used for the random number noise generator (creating stochastic Langevin force $L_{\mathrm{t}}$ in Eq. 2), although the dwell time mean values $\Delta_{\mathrm{m}}$, and standard deviations $\sigma$ (see Sect.3.2) remain unchanged.

For comparison with experimental results of Zhou et al. (2004) and Gao et al. (2006a,b,c) only simulations like those of Fig. 6, however without initial stimulus-off $(\mu=0)$ phase are evaluated. The reported experiments were performed with subjects viewing an ambiguous display, e.g., with a Necker cube, and pressing a button for indicating the time of conscious percept reversals $\mathrm{P} 1 \Leftrightarrow \mathrm{P} 2$.

A typical time series of simulated dwell times is shown in Fig. 8, indicating percept switching $\mathrm{P} 1 \Rightarrow \mathrm{P} 2$.

In order to obtain a sufficiently large number of dwell time intervals all evaluations are based on 100 successive simulations of $5000 T_{\mathrm{S}}=100 \mathrm{~s}$ (nominal simulation time interval $T_{\mathrm{S}}=20 \mathrm{~ms}$ ). Dwell times were reliably determined by evaluating zero crossings between $\mathrm{P} 1$ and $\mathrm{P} 2$ stationary $v$-levels after subtraction of a threshold value $v=1.7$ from the smoothed time series, using a self-written Matlab code. For smoothing, the high frequency limit cycle and chaotic oscillations (e.g., eigenfrequency spectrum according to Eq. 5), low pass filtering of original time series was performed by applying three iterations of a standard digital filter (Matlab function "filter(b, a, X)", with feedforward coefficient vector $b$ of dimension (windowsize) 80 , feedback coefficient $a=1, X=$ data vector containing all $(>500,000)$ $v$-values of the simulated time series). The small number of incorrectly determined P1-P2 reversals due to remaining large P2 fluctuations were eliminated by ignoring dwell times $<10$ sample intervals $T_{\mathrm{S}}(\Delta<200 \mathrm{~ms})$. This procedure ensured that no significant deviation of the dwell time statistics (relative frequency) from the $\Gamma$-density (see Sects. 1.4 and 3.2) at small perceptual duration times was created by
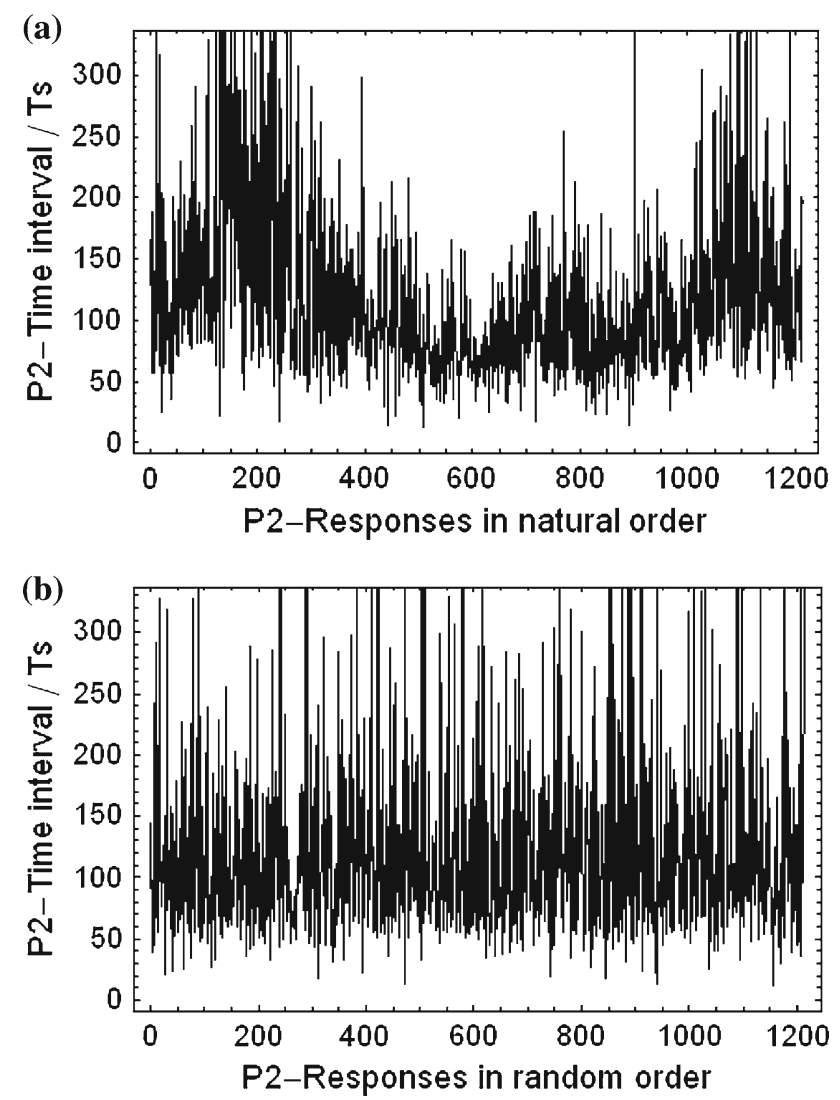

Fig. 8 Time series of percept duration intervals (dwell times) $\Delta(\mathrm{P} 2)$. a Simulated are the experimental button presses in natural order for transition between perceived (stationary) states $\mathrm{P} 1 \Rightarrow \mathrm{P} 2$ in Fig. 3. b Randomized order of time interval values exhibiting white noise (random walk process) with the same variance $\mathrm{s}^{2}$ as in $\mathbf{a}$

the high frequency high amplitude fluctuations as depicted in Fig. 6a.

The simulated $\Delta(\mathrm{P} 2)$-duration time series in Fig. $8 \mathrm{a}$ as well as the randomized version in Fig. $8 \mathrm{~b}$ reproduce qualitatively the experimentally obtained results of Gao et al. (2006a,b,c). Whereas Fig. 8a clearly exhibits some kind of structure within the time series, the randomized data in Fig. $8 \mathrm{~b}$ show white noise with the same variance, however constant power spectral density. Only $\Delta(\mathrm{P} 2)$-intervals are shown due to the $\mathrm{P} 1-\mathrm{P} 2$ asymmetry (mean values $\Delta_{\mathrm{m}}(\mathrm{P} 1) \neq$ $\left.\Delta_{\mathrm{m}}(\mathrm{P} 2)\right)$ mentioned before, and discussed as follows.

\subsection{Dwell time distribution and long range correlations}

The numerical simulations are analyzed with respect to the relative frequency of perceptual duration times $\Delta(\mathrm{P} 1), \Delta(\mathrm{P} 2)$ and to long range correlations. The dwell time statistics (relative frequencies) was shown in numerous experimental investigations (e.g., Levelt 1967; Borsellino et al. 1972; Zhou et al. 2004; Nakatani and van Leeuwen 2005, 2006) and theoretical modeling approaches (e.g., Ditzinger and Haken 1989; 
Kelso et al. 1995; Natsuki et al. 2000; Fürstenau 2006) to correspond to the density of a gamma $(\Gamma)$-distribution as a reasonable approximation with shape parameter $\alpha$ and scaling parameter $\lambda$.

$f(\Delta)=\lambda^{\alpha} \frac{\Delta^{\alpha-1}}{\Gamma(\alpha)} \exp \{-\lambda \Delta\}$

with gamma function $\Gamma(\alpha)$. Mean and variance are given by $\Delta_{\mathrm{m}}=\alpha / \lambda$ and $\sigma^{2}=\alpha / \lambda^{2}$, respectively, and coefficient of variation $c v=\sigma / \Delta_{\mathrm{m}}=1 / \sqrt{ } \alpha$ which is independent of the mean (e.g., Dodson and Scharcanski 2003).

As mentioned in the Sect. 1.4, the nonlinear time series analysis in this study is based on the determination of the self-similarity or Hurst parameter $H(=0 \ldots 1)$ which is an established indicator of the fractal content or long range correlations (Mandelbrot 1991; Beran 1992). $H=0.5$ represents the random process (random walk or Brownian motion). Because all of the usually employed methods require very long time series for determination of reliable $H$-value which very often are not available, different methods have been developed which may be used for critical comparison and consistency checks of the resulting $H$-estimates. Here, we use three different methods which after critical discussion in Sect. 4 lead to a value in good agreement with the experimental results of Gao et al. (2006a).

\subsubsection{Relative frequency of perceptual dwell times}

A theoretical motivation for focusing on the $\Gamma$-density as fitting function for the relative frequencies of dwell times is provided by recent study of Dodson and Scharcanski (2003). They outline an information theoretic differential geometry of the two-parametric family of $\Gamma$-distributions which contains the exponential distribution $(\alpha=1)$ as a special case. The results indicate this family to have a natural role in representing departures from a random process. $\alpha=1$ represents the Poisson process with maximum information entropy which was often used to model waiting times in distributed communication networks. Larger $\alpha$-values correspond to smoothing or temporal dispersion of events having a lower variance than Poisson. A gamma-density with integer $\alpha$ is the $\alpha$-fold convolution of the exponential. Because in experiments often (approximately) integer $\alpha$-values (typically $2,3,4$ ) are observed several authors assume this as an indicator for a discrete stochastic multistep process with $\alpha$ successive discrete cognitive states (e.g., Murata et al. 2003).

The experimental literature as well as theoretical considerations (Levelt 1967) often yield $c v=\sigma / \Delta_{\mathrm{m}}=1 / \sqrt{ } \alpha \approx 0.5$, however, with large experimental inter-individual scattering of mean values of $\alpha$ between 2 and $5 \mathrm{~s}$ (e.g., Borsellino et al. 1972). Of course, this also depends on the detailed experimental conditions. For the present theoretical model,
Table $1 \Gamma$-distribution parameters of simulated percept duration time series $\Delta(\mathrm{P} 2)$ as obtained with $v_{\mathrm{b} 0}=v_{\text {be }}$ and different memory/learning time constants $\tau_{\mathrm{M}}, \tau_{\mathrm{L}}$ for $\mu=0.6, \gamma=60, \tau_{\mathrm{G}}=500, T=2, \tau=$ $0.5, J_{\omega}=0.004$ : listed are shape parameter $\alpha$, P2-duration mean $\Delta_{\mathrm{m}}$ (in seconds), and relative standard deviation of $\Gamma$-distribution fit to relative $\Delta_{\mathrm{m}}$ frequencies

\begin{tabular}{rrrll}
\hline$\tau_{\mathrm{M}}$ & \multicolumn{1}{l}{$\tau_{\mathrm{L}}$} & $\alpha( \pm \delta \alpha)$ & $\Delta_{\mathrm{m} / \mathrm{s}}$ & $\sigma / \Delta_{\mathrm{m}}$ \\
\hline 1,000 & 2,000 & $3.5(0.2)$ & 2.7 & 0.53 \\
1,000 & 100,000 & $2.2(0.2)$ & 2.7 & 0.67 \\
2,000 & 100,000 & $3.1(0.2)$ & 2.5 & 0.57 \\
3,000 & 100,000 & $3.6(0.3)$ & 2.4 & 0.53 \\
10,000 & 100,000 & $4.3(0.3)$ & 2.4 & 0.48 \\
50,000 & 100,000 & $4.4(0.3)$ & 2.6 & 0.48 \\
\hline
\end{tabular}

$\delta \alpha=95 \%$ confidence intervals

these observations were reproduced in previous publications (Fürstenau 2004, 2006, 2007).

As a new result Table 1 lists the data of a series of simulations with varying memory time constant $\tau_{\mathrm{M}}$. Initial results were provided already in Fürstenau (2007). Average shape parameters $\alpha$ and average perceptual duration times $\Delta_{m}$ are listed of 100 simulation runs with $N=5000$ time steps $T_{\mathrm{S}}$ each $\left(=100 \mathrm{~s}\right.$ for $\left.T_{\mathrm{S}}=T / 2=20 \mathrm{~ms}\right)$ and parameters as those used for Fig. 6 (Sect. 3.1). For the stochastic Langevin force $L_{\mathrm{t}}$ in Eq. 2, a noise power spectral density $J_{\omega}=0.004$ is required to match the ratio $\sigma / \Delta_{\mathrm{m}} \approx 0.5$, with the given delay and time constants.

For a simulation run with averaging over 100 time series mean values $\Delta_{\mathrm{m}}(\mathrm{P} 2)=2-3 \mathrm{~s}$ and coefficient of variation $\mathrm{cv}=\sigma / \Delta_{\mathrm{m}}=1 / \sqrt{ } \alpha \approx 0.5-0.6$ are observed which are within the range of the above-mentioned experimental findings. The magnitude of $\Delta_{\mathrm{m}}$ for $\mu=0.6$ is about 2-3 times the analytical estimate of Eq. 6 with $\mu=0.2$, near the node bifurcation threshold $\mu=0.18$. Simulation with low contrast value $\mu=0.23$ without noise yields percept reversal frequency $f=48 \min ^{-1}\left(\Delta_{\mathrm{m}}\left(P_{\mathrm{i}}\right)=0.6 \mathrm{~s}\right)$ in reasonable agreement with the analytical estimate for $\mu=0.2, f_{\mathrm{D}}=33 \min ^{-1}\left(\Delta_{\mathrm{D}}=0.9 \mathrm{~s}\right)$ as obtained via Eq. 6 with zero noise. While mean values $\Delta_{\mathrm{m}}$ decrease with contrast parameter $\mu$ and are determined basically by the fatigue time constant $\gamma=60 T_{\mathrm{S}}=1.2 \mathrm{~s}$, the ratio $\sigma / \Delta_{\mathrm{m}} \approx 0.5$ requires a suitable choice of the Langevin force $L_{\mathrm{t}}$ with attention noise spectrum $J_{\omega}$ and noise sample time $t_{\mathrm{c}}$, which may be compared with the speed of attention-parameter variation $\Delta G / \Delta t$ (typically $1.5 / 250 T_{S} \approx 0.3 \mathrm{~s}^{-1}$ ). This has the same order of magnitude as the standard deviation $s$ estimated via the covariance $J_{\omega} / t_{\mathrm{c}}=s^{2}=0.04$. With zero noise $\left(J_{\omega}=0\right.$, all other parameters unchanged) simulations yield periodic $\mathrm{P} 1-\mathrm{P} 2$ self-oscillations with purely deterministic superimposed limit cycle oscillation exhibiting a percept reversal frequency $f=20 \min ^{-1}\left(\Delta_{\mathrm{m}}\left(P_{\mathrm{i}}\right)=1.5 \mathrm{~s}\right)$ which increases to the above-mentioned $48 \mathrm{~min}^{-1}$ for $\mu=0.23$. A 
purely periodic $\mathrm{P} 1 \Leftrightarrow \mathrm{P} 2$ self-oscillation for the zero noise case $\left(J_{\omega}=0\right)$ was shown already with the dynamical models of Ditzinger and Haken (1989) and Wilson (1999).

For $\tau_{\mathrm{M}, \mathrm{L}}>50,000$, the shape parameters $\alpha$, mean percept durations $\Delta_{\mathrm{m}}$, and standard deviations $\sigma$ converge to those of the previously reported simulations without memory and learning (Fürstenau 2006, 2007), i.e., with constant perception bias $v_{\mathrm{b}}$. Large $\tau_{\mathrm{L}, \mathrm{M}}$ reduces the memory and learning effects and represents quasi static preference ratio. Table 1 indicates a slightly increasing coefficient of variation with decreasing time constant $\tau_{\mathrm{M}}$, starting at $\mathrm{cv}=$ $\sigma / \Delta_{\mathrm{m}} \approx 0.5(\alpha=4)$ for the memoryless condition (large $\left.\tau_{\mathrm{M}}\right)$. This corresponds to increasing long range correlations due to growing influence of deterministic as compared to stochastic (attention noise) contributions (see below).

\subsubsection{Long range correlations}

Figure $9 \mathrm{a}$ and $\mathrm{b}$ shows the autocorrelation functions $(\mathrm{ACF})$ obtained from a time series like that one of Fig. 8 for $\tau_{\mathrm{M}}=$ $3,000 T_{\mathrm{S}}$ and for $\tau_{\mathrm{M}}=100,000$ (i.e., negligible influence of memory effects), respectively.

While the mean ACF of Fig. 9a appears significantly shifted toward positive values with indication of weak oscillation, the randomized case of Fig. 9b exhibits the expected zero correlation corresponding to white gaussian noise. The weak shift of the original reversal time series ACF is a first indication of long range correlations (see next section, Fig. 13). This was shown by Gao et al. (2006a) with the same kind of ACF for their experimental data resulting in a quantitatively surprising agreement with our simulation, even with regard to the oscillatory features.

For second-order self-similar or fractal time series $X=$ $\left(X_{i}, i=0,1,2, \ldots\right)$, the autocorrelation function is independent on the time $t_{\mathrm{i}}$ and depends only on the time difference (Gao et al. 2007). Second-order stationarity was verified for the time series underlying Fig. 9 by calculating ACF's for 10 non-overlapping data blocks and checking for the independence on time, in this way indicating dependence on time lag $k=i-j$ only.

In what follows, it will be shown that with the present model the coupling of the preference parameter (attention bias) $v_{\mathrm{b}}$ to the perception state leads to the long range correlations via memory effects. They are quantified by the Hurst parameter $H$. Values within the range $0.5<H<1$ characterize so-called second-order self-similar processes with persistent correlation. Quantitative estimates of $H$ may be obtained in different ways. Gao et al. (2006a) used the popular dependence of the variance of the dwell-time time series $\left\{\Delta_{i}, i=1,2, \ldots, n\right\}$ on the number of data points $m$ used for averaging non-overlapping blocks of size $m$ of the original data (variance-time (VT) method; e.g., Beran 1992; Gao et al. 2006b), via
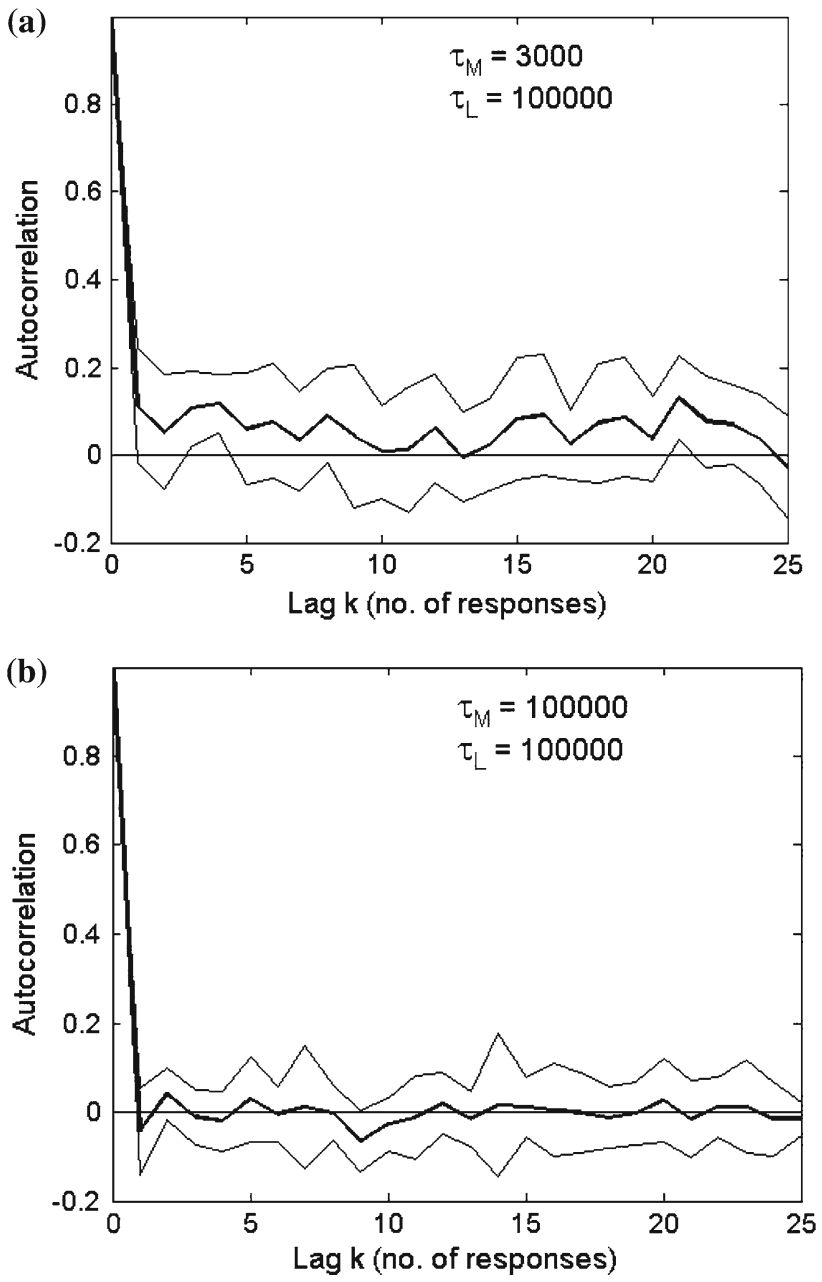

Fig. 9 Autocorrelation functions calculated from P2-dwell times of reversal time series of Fig. 6 a. Simulation parameters $\mu=0.6, v_{\mathrm{be}}=$ $1.5, T=2, \tau=0.5, \gamma=60, \tau_{\mathrm{G}}=500, J_{\omega}=0.004$ with sample time $t_{\mathrm{c}}=0.1$ (time units $T_{\mathrm{S}}=T / 2=20 \mathrm{~ms}$ ). Thick and thin lines representing mean and standard deviation respectively of 10 autocorrelation functions using succeeding sections of length $5000 T_{\mathrm{S}}$ of the whole time series. a Simulation with memory time constant $\tau_{\mathrm{M}}=3000 T_{\mathrm{S}}$ indicating long range dependence (average value of ACF > 0). b Simulation with $\tau_{\mathrm{M}}=100,000 T_{\mathrm{S}}$ indicating zero correlation

$\operatorname{var}\left(\Delta_{\mathrm{t}}^{(\mathrm{m})}\right)=\sigma^{2} m^{2 \mathrm{H}-2}$

with variance $\sigma^{2}=\operatorname{var}\left(\Delta_{\mathrm{t}}^{(1)}\right)$. This relationship is valid for exactly second-order self-similar processes and for $\lim m \rightarrow$ $\infty$. In Gao et al. (2006b), the authors investigate multifractal processes and show the equivalence of Eq. 8 with the fluctuation analysis (FA) of a random walk process derived from a zero mean fractional Gaussian noise process. They discuss, in detail, the validity range of this and related methods which is particularly important near $H=0$ and 1 . These aspects of the analysis will be discussed in Sect. 4. Equation 8 provides an estimate of $H$ via the slope $\beta$ of the $\log$ (variance $(\Delta, m))$ versus $\log ($ sample size $m)$ plot through the relation 

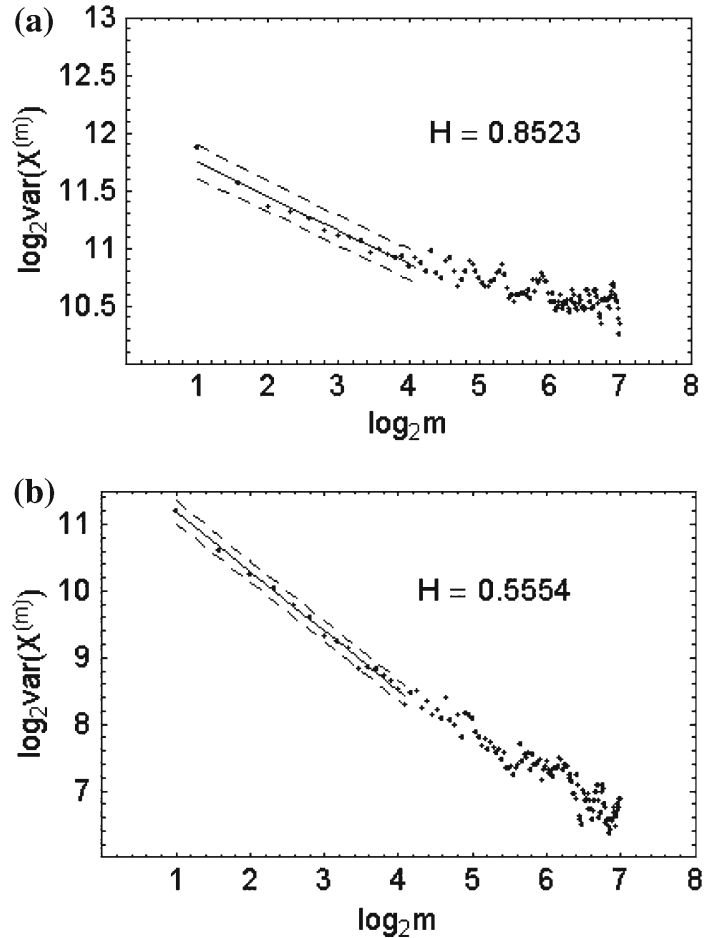

Fig. 10 Variance-time analysis. Plot of $\log$ (variance) versus $\log$ (sample size $\mathrm{m})$ of $\Delta(\mathrm{P} 2)$ for $\mathrm{P} 2$-dwell times as obtained from simulations with 100 runs of $5000 T_{\mathrm{S}}$ each. Simulation parameters like in Fig. 8a. Linear fit (straight line; dotted lines $=95 \%$ confidence intervals for predicted responses of single observations) for estimating $H$ via the slope of the $\log$ (variance) $-\log$ (time) plot. a Attention bias (preference) time constants $\tau_{\mathrm{M}}=3000, \tau_{\mathrm{L}}=100,000$, evaluation of 1,276 discrete percept intervals. b No significant memory and learning effect $(H \approx 0.5$, corresponding to random walk) with $\tau_{\mathrm{M}}=100,000, \tau_{\mathrm{L}}=100,000$, evaluation of 1,608 discrete percept intervals

$H=1-\beta / 2$. Figure $10 \mathrm{a}$ and $\mathrm{b}$ depicts this VT plot with $\mathrm{a}$ sample size range $m=1-7$ as used by Gao et al. (2006a). Simulations are evaluated for the two cases with and without significant memory effect, expressed by the memory time constants $\tau_{\mathrm{M}}=3,000$ and $\tau_{\mathrm{M}}=100,000$, respectively.

In Fig. 10a, the variance $(\Delta, m)$ of the series of 1,276 perceptual duration times $\Delta$, splitted into sections of length $m$ for averaging, is plotted versus the sample size $m$ in double logarithmic scale. The $H$ parameter is determined from the slope $\beta$ by fitting a straight regression line to the data (with $\left.\log _{2}(m) \leq 7\right)$ using the sample range $\log _{2}(m) \leq 4$, yielding $H=0.85( \pm 0.01)$. The fit (solid line) includes $95 \%$ confidence intervals (dotted line) of parameter estimates. Only variance values up to averaging sample size $\log _{2}(m)=4$ was used due to the decreasing statistical reliability with decreasing number of averaged time series data with increasing $m$. For memory time constant $\tau_{\mathrm{M}}=3000 T_{\mathrm{S}}=60 \mathrm{~s}$ and learning time constant $\tau_{\mathrm{L}}=100,000$ (which is sufficiently large to show no significant effect), a significant long range correlation is observed due to the (short term) memory effect.
Table 2 Hurst parameters of simulated perceptual duration time series $\Delta(\mathrm{P} 2)$ as obtained with initial value $\mathrm{v}_{\mathrm{b} 0}=\mathrm{v}_{\mathrm{be}}=1.5$ and different memory / learning time constants $\tau_{\mathrm{M}}, \tau_{\mathrm{L}}$ for $\mu=0.6, \gamma=60, \tau_{\mathrm{G}}=$ $500, \mathrm{~T}=2, \tau=0.5, \mathrm{~J}_{\omega}=0.004 . \delta H=95 \%$ confidence intervals

\begin{tabular}{cccc}
\hline$\tau_{\mathrm{M}}$ & $\tau_{\mathrm{L}}$ & $H_{\mathrm{KG}}( \pm \delta H)$ & $H_{\mathrm{var}}( \pm \delta H)$ \\
\hline 1,000 & 2,000 & $0.65(0.07)$ & $0.79(0.02)$ \\
1,000 & 100,000 & $0.75(0.08)$ & $0.86(0.02)$ \\
2,000 & 100,000 & $0.71(0.07)$ & $0.88(0.02)$ \\
3,000 & 100,000 & $0.68(0.07)$ & $0.85(0.02)$ \\
10,000 & 100,000 & $0.58(0.07)$ & $0.73(0.02)$ \\
50,000 & 100,000 & $0.51(0.06)$ & $0.58(0.02)$ \\
100,000 & 100,000 & $0.49(0.06)$ & $0.56(0.02)$ \\
\hline
\end{tabular}

In addition to the statistical uncertainty of $\delta H \approx 0.02$ corresponding to the $95 \%$-confidence interval of parameter estimates, a systematic error of the $H$-values obtained from the slope within the $\log _{2}(m)=1-4$ range has to be assumed. Reason is the limes condition (large $m$ ) for the validity of Eq. 8 which suggests to use the larger $m$-range for determining the slope value $\beta$. For estimating the order of magnitude of this systematic error, also the range $\log _{2}(m)=3-6$ was used for fitting a straight line to the data, yielding $H=$ $0.93 \pm 0.02$ due to the lower slope value, as can be guessed from Fig. 10a.

The memory time constant $\tau_{M}$ as origin of long range correlations is demonstrated with Fig. 10b where $\tau_{\mathrm{M}}=100,000$ is selected (vanishing memory effect), yielding $H \approx 0.5$ within the given uncertainties, i.e., a random walk process. A set of $H$-values for memory time constants $\tau_{\mathrm{M}}$ between $\tau_{\mathrm{M}}=1000 T_{\mathrm{S}}=20 \mathrm{~s}$ and $100,000 T_{\mathrm{S}}=33 \mathrm{~min}$ is shown in the last column of Table 2.

For constant learning time constant $\tau_{\mathrm{L}}=100,000 T_{\mathrm{S}}$, it indicates the increasing long range correlations with decreasing $\tau_{\mathrm{M}}$, starting from a random noise process for $\tau_{\mathrm{M}}>$ $50,000 T_{\mathrm{S}}=16 \mathrm{~min}$. The magnitude of the systematic error to be considered with the VT method is indicated using the $\log _{2}(m)=3-6$ range for the linear regression. Compared to the values of Table 2 , the first value $H_{\mathrm{var}}\left(\tau_{\mathrm{M}}=1,000, \tau_{\mathrm{L}}=\right.$ $2,000)$ is smaller $(0.69)$, the next two values are the same (within the standard error), whereas the last four values are significantly larger than those with the $\log _{2}(m)=1-4$ range.

As an alternative for estimating $H$, the method of Kettani and Gubner (2006) (KG-method) is employed which also applies to second-order self-similar or fractional Gaussian processes. It estimates $H$ via the lag-1 $\mathrm{ACF} \rho(1)=2^{2 \mathrm{H}-1}-1$ and for sample size $n$ also provides an upper boundary of the $95 \%$ confidence interval

$\hat{H}_{n} \pm \delta \hat{H}_{n}=0.5\left[1+\log _{2}\left(\hat{\rho}_{n}(1)\right)\right] \pm 5 / \sqrt{n}$. 
The authors show that for second-order self-similar or fractional ARIMA processes their method yields more reliable results (smaller confidence interval) as compared to the widely used wavelet method (e.g., Gao et al. 2007). Table 2 lists in column 3 , the $H_{\mathrm{KG}}$ values of the $\Delta(\mathrm{P} 2)$ time series for memory and learning time constants $\tau_{\mathrm{M}}, \tau_{\mathrm{L}}$ between $1,000 T_{\mathrm{S}}$ and $100,000 T_{\mathrm{S}}$. For constant $\tau_{\mathrm{L}}=100,000$, the $\mathrm{H}_{\mathrm{KG}}$-values increase from 0.5 (random noise) to 0.75 with $\tau_{\mathrm{M}}$ decreasing from $16 \mathrm{~min}$ to $20 \mathrm{~s}$, again indicating significant long range correlations, although significantly smaller than those obtained with the VT method. Large $\tau_{\mathrm{L}, \mathrm{M}}$ values $>10,000$ again reduce the memory and learning effects approaching quasi static preference parameter $v_{\mathrm{b}}\left(\mathrm{d} v_{\mathrm{b}} / \mathrm{d} t \approx 0\right)$ with increasing time constant. Correspondingly, the last rows of the table exhibits vanishing long range correlations $(H \approx 0.5)$. All simulations, however, confirm significant long range correlations with $H$ increasing when $\tau_{\mathrm{M}}$ decreases (memory effect increases). The learning effect ( $\tau_{\mathrm{L}}$ ) exhibits only weak influence on $H$, compared to $\tau_{\mathrm{M}}$ because the former only acts in the beginning of the time series.

For both methods, significant long range correlations $(H>0.5)$ are observed due to the attention bias dynamics if the memory time constant $\tau_{\mathrm{M}}<50,000 T_{\mathrm{S}}=100 \mathrm{~s}$. In addition the learning component of $\mathrm{d} v_{\mathrm{b}} / \mathrm{d} t$ influences the dynamics in the initial phase if $\left|v_{\mathrm{be}}-v_{\mathrm{b}}(t=0)\right|>0(M$ can switch from 0 to 1 ), but only for sufficiently small $\tau_{\mathrm{L}}$, typically $<2,000$. If $v_{\mathrm{be}}=v_{\mathrm{b} 0}=v_{\mathrm{b}}(t=0)$ the bias $v_{\mathrm{b}}$ corresponds to its preset equilibrium value $v_{\text {be }}$ and learning stops whereas the dynamic memory variation continues due to coupling to $v_{\mathrm{t}}$.

The third method used to derive an estimate of the Hurst parameter uses the power spectral density of the dwell time series which is related to the variance-time method (Eq. 8) via the Wiener-Khinchine theorem (Beran 1992; Gao et al. 2007). A $1 / f^{\alpha}$ characteristic is predicted in the limit of low frequencies $\mathrm{f}$

$\mathrm{PSD}=f^{1-2 \mathrm{H}}$.

Figure 11a and $\mathrm{b}$ shows in double-logarithmic scale the frequency characteristics for $\tau_{\mathrm{M}}=3,000$ (significant memory effects) and $\tau_{\mathrm{M}}=100,000$ (negligible memory effects), respectively, $\left(\tau_{\mathrm{L}}=100,000\right.$ in both cases) obtained by evaluating a single long time series of $50,000 T_{\mathrm{S}}$ yielding about 850 percept reversals as automatically determined with the threshold detection code mentioned above (Sect.3.1). The dwell-time series for spectral analysis is derived by equidistant sampling of the percept series at time intervals $\Delta t=5 T_{\mathrm{S}}=100 \mathrm{~ms}$ yielding $10,000 \Delta t$ samples for calculating the power spectral density (PSD) via fast Fourier transform (using Mathematica 5.2 time series toolbox). Minimum and maximum frequencies are $\omega_{\min }=210^{-4} \mathrm{rad} / \Delta t$ and $\pi \mathrm{rad} / \Delta t$, corresponding to a full wave extending over
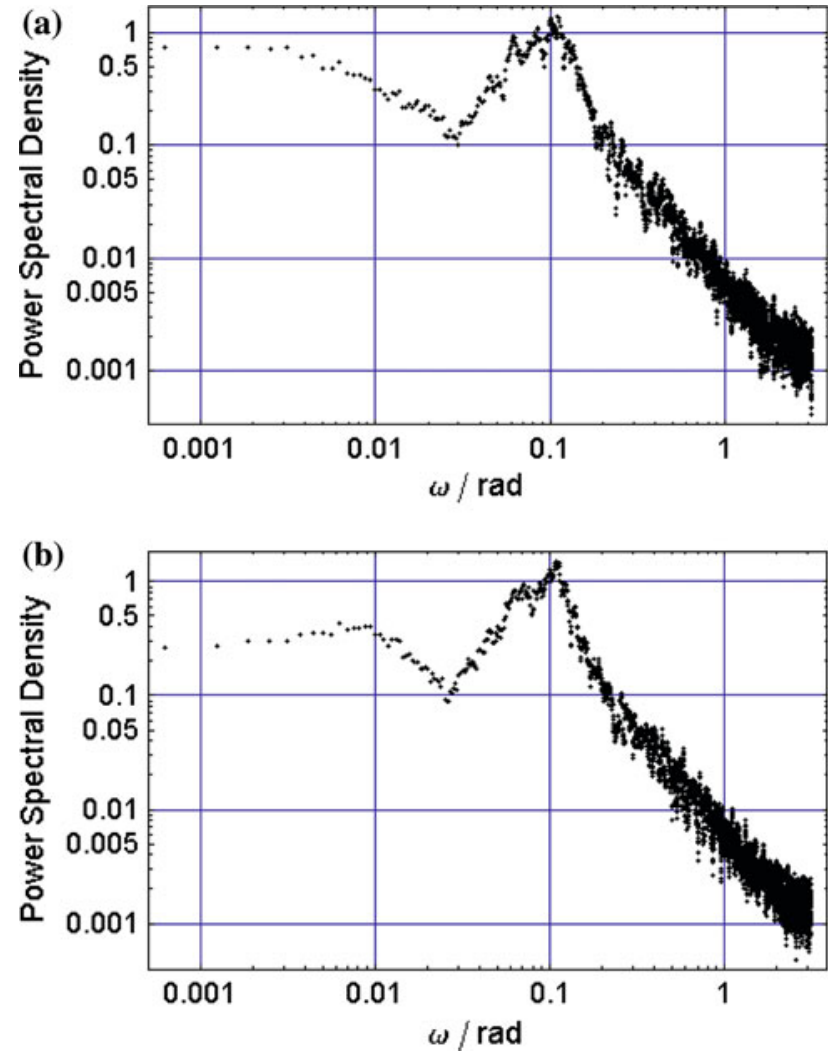

Fig. 11 Power spectral densities up to $\omega=\pi / \Delta t$, with sampling interval $\Delta t=5 T_{\mathrm{S}}$, of percept duration time series for $\mathbf{a} \tau_{\mathrm{M}}=3000$ and b $\tau_{\mathrm{M}}=100$, 000. Global reversal rate maximum due to deterministic dynamics of Eq. $1-3$ at $\omega=0.1 \mathrm{rad} / \Delta t$

the whole time series $N=10,000 \Delta t$ and a minimum period of $2 \Delta t$, respectively. The spectrum exhibits four characteristic sections which may be attributed to different origin.

The PSD's in Fig. 11a and b exhibit significant differences in the low frequency range $\omega<0.01 \mathrm{rad} / \Delta t$. A more or less linearly decreasing section in the double log scale, representing roughly $1 / f^{\alpha}$ characteristic, can be seen between 0.003 and $0.03 \mathrm{rad} / \Delta t$ in Fig. $11 \mathrm{a}$. The difference between the two spectra $\mathrm{a}$ and $\mathrm{b}$ for $\omega<0.02 \mathrm{rad} / \Delta t$ may be attributed to the memory effect-induced through the short time constant $\tau_{\mathrm{M}}=3,000$. A broad global frequency peak with several local maxima is observed around $0.1 \mathrm{rad} / \Delta t$, with a decrease with roughly $1 / f$ characteristic up to the high frequency limit at $\pi \mathrm{rad} / \Delta t$ which is not of interest here. The peaks at $\omega \approx 0.06$ and $0.1 \mathrm{rad} / \Delta t$ correspond to the full periods of percept reversals $2 \Delta_{\mathrm{m}}(\mathrm{P} 1) \approx 2 \pi 0.1 \mathrm{~s} / 0.07=9 \mathrm{~s}$ and $2 \Delta_{\mathrm{m}}(\mathrm{P} 2) \approx 6 \mathrm{~s}$. As expected mean reversal rates of $1 / \Delta_{\mathrm{m}}=$ $13 \ldots 20$ transitions $\mathrm{P} 1 \Leftrightarrow \mathrm{P} 2 \min ^{-1}\left(\Delta_{\mathrm{m}}=3-4.5 \mathrm{~s}\right)$ are observed which again appear to be consistent with the analytical approximation of Eq. 6, and which are reasonable agreement with the usual experimental findings (e.g., Borsellino et al. 1972; De Marco et al. 1977; Zhou et al. 2004).

From the PSD's of Fig. 11, only the range between $0.003 \mathrm{rad} / \Delta t$ (corresponding to a time interval of $200 \mathrm{~s}$ or 

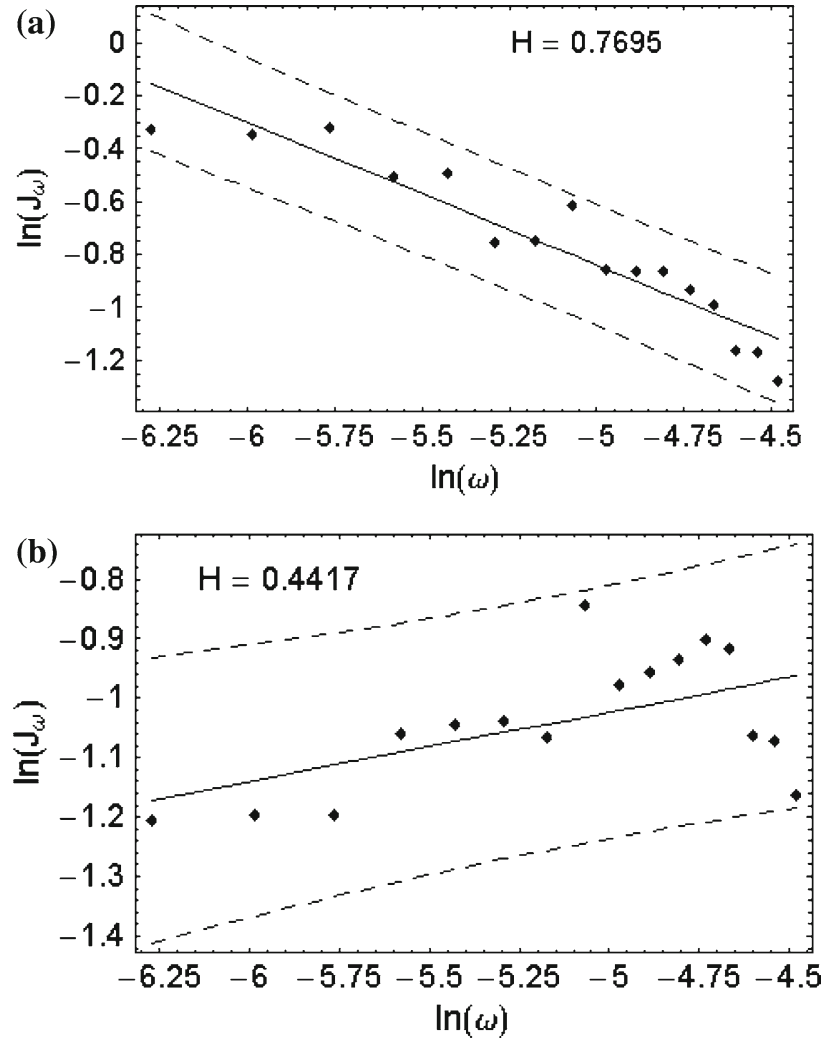

Fig. 12 Log- $\log$ plots of power spectral density of percept duration time series from frequency interval $\omega=0.003-0.012 \mathrm{rad} / \Delta \mathrm{t}$ of Fig. 11. a $\tau_{\mathrm{M}}=3000$ : PSD exhibits decreasing general trend which is fitted via linear regression corresponding to $1 / f^{\alpha}$-characteristic (Eq. 11) yielding Hurst parameter $H \approx 0.8$. Dotted lines indicate $95 \%$ confidence intervals of predicted responses of single observations. b Negligible memory effect with $\tau_{\mathrm{M}}=100,000$ yielding $H \approx 0.5$ (random walk)

ca. 50 average percept intervals $\Delta_{\mathrm{m}}$ ) and $0.012 \mathrm{rad} / \Delta t$ (or ca. $5 \Delta_{\mathrm{m}}$ ) is evaluated for determining the long range correlations via Eq. 10 using $H=(1-\beta) / 2$, with $\beta=$ slope in the double-logarithmic plot of the selected part of the PSD. Like Gao et al. (2006a), we take a linear fit to the log-log plot of this PSD interval as indicative of the $1 / f^{\alpha}$ noise characteristic of the process and obtain the results shown in Fig. 12 $\mathrm{a}$ and $\mathrm{b}$.

Equation 10 is valid in the limit of low frequencies (Beran 1992; corresponding to the large sample size limit of the variance-time method; Eq. 8). Due to the long time series of $50000 T_{\mathrm{S}}$ this condition more or less appears to be fulfilled, so that the $H$-estimate as obtained via the slope of the linear fit to the data $(-0.29 \pm 15 \%$ standard error) does not suffer the systematic error of the VT-method. On the other hand, the fit covers only a the small frequency range, and it probably is a lower limit because the slope obtained by the linear regression is increased by the rather broad frequency peak of the selfoscillator reversal rate $1 / \Delta_{\mathrm{m}}$ in the PSD shown in Fig. 11 . The $H$-value obtained via the PSD $(H=0.77 \pm 15 \%)$ for $\tau_{\mathrm{M}}=3,000 ; H=0.44$ for $\tau_{\mathrm{M}}=100,000$ lies between the corresponding values $H_{\mathrm{KG}}, H_{\mathrm{var}}$ in Table 2, and is in good agreement with the experimental values presented by Gao et al. (2006a,b,c). Their spectral range for the dwell times between switches $\ln (f)=-3.4, \ldots,+0.6$, corresponding to full reversal periods (between $\mathrm{P} 2$-percepts in our case) $\ln (\omega) \approx \ln (f)+\ln (2 \pi)-\ln (2)=(-3.4, \ldots,+0.6)+$ $1.84-0.7=-2.3, \ldots,+1.7$ covers a larger interval than ours. The range in our simulations extends to lower frequency values $(-6.25)$ due to the long time series of $50000 T_{\mathrm{S}}=$ $17 \mathrm{~min}$, however, is clipped also at a lower high frequency value $(-4.5$ instead of +1.7$)$ to prevent any distortion from the large $2 \Delta_{\mathrm{m}}$-peak with $\Delta_{\mathrm{m}} \approx 2.5-3 \mathrm{~s}$.

\section{Discussion}

The present minimum architecture of a nonlinear dynamics model for simulating bistable perception via mapping to basic thalamocortical reentrant loops yields a number of time series characteristics in good quantitative agreement with experimental results (for references see Sect.1).The linearized analytical approximation (Eq. 6) reveals the self-oscillator dynamics as basic mechanism by deriving the correct order of magnitude of the percept reversal eigenfrequency for small stimulus parameter value (difference-of-meaning) $\mu$, with fatigue time constant $\gamma=60 T_{\mathrm{S}}=1.2 \mathrm{~s}$, feedback delay $T=2 T_{\mathrm{S}}=40 \mathrm{~ms}$, and low-pass filter time constant $\tau=1 T_{\mathrm{S}}$.

Previous modeling approaches reported in the literature often focused on reproducing the two-parametric gamma density of the dwell-time time series. As mentioned in Sect.3.2, this is motivated by its statistical properties as detailed in recent study of Dodson and Scharcanski (2003). It shows the shape parameter $\alpha$ to measure the deviation from the random (Poisson) process $(\alpha=1)$ with maximum information entropy. Experiments of Murata et al. (2003) provided support for the early proposal of integer shape parameter values (e.g., $\alpha=4$; Levelt 1967), indicating a multi-step $(\alpha=2,3,4)$ cognitive process. It corresponds to a stochastic clock generating exponentially distributed pulses and percept switches after each $\alpha$ th pulse (De Marco et al. 1977). Although, the $\alpha$-values of the present results are in good agreement with the typical values published in the literature $(a=2-4.5)$, they give no indication of integer values.

The present evaluation goes beyond the modeling of dwell time distributions by applying nonlinear time series analysis to the stochastic sequence of dwell times. It provides a new approach for evaluating and quantifying deviations from the random case (i.e., Poisson process with shape parameter $\alpha=1$ ) which were found experimentally by Gao et al. (2006a). The focus here is on the long range correlations or fractal character of time series as characterized by the selfsimilarity or Hurst parameter $H$ which is shown to be explicitly dependent on the memory time constant $\tau_{\mathrm{M}}$ of the PAM 
(perception-attention-memory) equations used for formalizing the model. The three methods used in this analysis for determining $H$-values provide consistent results concerning the $H$ dependence on memory time constant $\tau_{\mathrm{M}}$. They differ, however, with regard to the magnitude of the effect and have to be discussed with regard to problems mentioned in Gao et al. (2006b).

The Hurst parameter $H_{\mathrm{KG}}=0.6-0.75$ (for $t_{\mathrm{M}} \leq 3000$ ) as obtained with the Kettani-Gubner method is systematically smaller as compared to $H_{\mathrm{var}}(0.7-0.85)$ obtained with the variance-time (VT) method which was also used by Gao et al. (2006a) for the evaluation of their bistability experiments. The evaluation of the $f^{\alpha}$-process after equidistant sampling and Fourier transform of the dwell time series provides $H$-values in between the two other methods. As mentioned before the $H$-values obtained by the VT-method suffer a systematic error because they are valid only for the limit of large sample size $m$ whereas for reason of sufficient statistics the linear regression procedure was limited to $\log _{2}(m)=4$. No such problem exists for the evaluation of the $f^{\alpha}$-process because, in this case, large sample size corresponds to the low frequency limit. The power spectrum of the dwell time series could in fact be (only) evaluated in a low frequency range, providing significantly lower $H$-values. In the present analysis, I follow the recommendation of Gao et al. (2006b) and use the spectral method not alone but for cross-checking only. The results can be treated as upper limit due to the distortion of the spectrum by the broad mean reversal rate $\left(1 / \Delta_{\mathrm{m}}\right)$ frequency peak originating from the self-oscillator dynamics. The lowest values with $H \approx 0.7$ (memory time constant $\tau_{\mathrm{M}}=60 \mathrm{~s}$ ) are obtained with the method based on the lag-1 ACF (Kettani and Gubner 2006).

As an evaluation of ACF's of simulated perceptual duration time series revealed no significant time dependence (see Fig. 9), stationarity appears to be fulfilled as one condition for the applicability of the algorithms. However, all three methods are derived for second-order self-similar processes which might not be exactly fulfilled for the PAM-model time series. Therefore, it is of interest to also compare the autocorrelation functions obtained from the computer experiments with the ideal one for the long range-dependent process or fractional Gaussian noise which is the necessary and sufficient condition for the validity of the three methods used for determining $H$ (e.g., Gao et al. 2006b):

$r(k)=\frac{1}{2}\left[(k+1)^{2 \mathrm{H}}-2 k^{2 \mathrm{H}}+|k-1|^{2 \mathrm{H}}\right]$

Figure 13 depicts this ACF (lag k) for $H$ values between 0.5 (Brownian motion or random walk process) and $1(100 \%$ correlation).

By comparison with Fig. 9a, it can be seen that a $H$-value between 0.65 and 0.7 exhibits the best agreement with the simulation results. A formal estimate may be obtained by

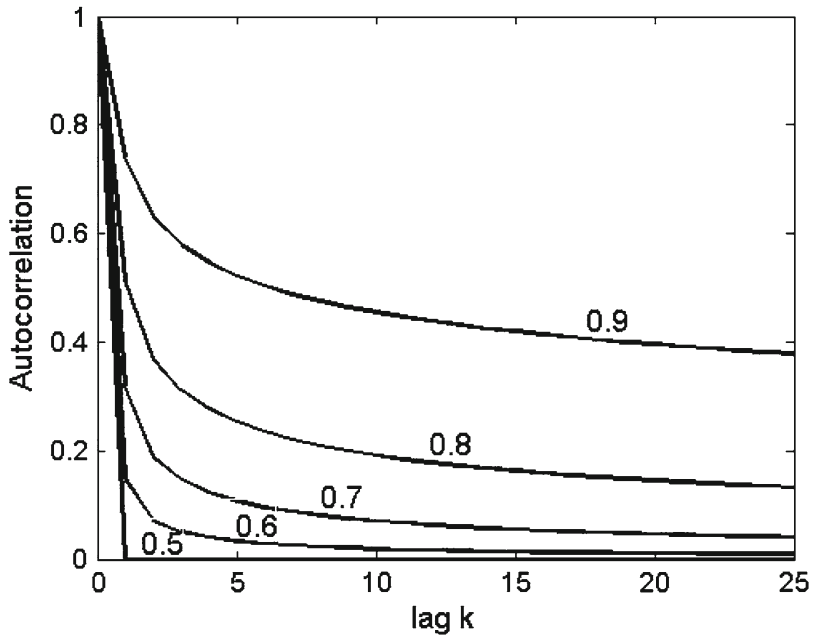

Fig. 13 Autocorrelation function of fractional Gaussian noise (Eq. 11) for different values of Hurst parameter $H$, as necessary and sufficient condition for the validity of the variance-time method (Eq. 8). Curves may be compared with simulation results of Fig. 9 for a $H$ estimate: $H \approx 0.65-0.7$

means of a nonlinear least squares fit and utilizing the approximation to Eq. 11 for large $k$ (Gao et al. 2007):

$r(k)=H(2 H-1) k^{2 \mathrm{H}-2}$.

Using the Matlab function nonlinear fit (X, y, fun, $b_{0}$ ) (with $y=$ vector of dependent variables (i.e., $k=1-25 \mathrm{AC}$ values), $\mathrm{X}=$ design matrix of independent variables (i.e., $k=$ $1-25)$, fun $=$ model function (Eq. 12), $b_{0}=$ initial estimate of the parameter vector (i.e., $\left.H_{0}\right)$ ), we obtain $H=0.65 \pm$ 0.03 (with a $95 \%$-confidence interval $\delta H / H=5 \%$ ) which appears to be an additional confirmation of our $H_{\mathrm{KG}}$-result. This means that $H_{\mathrm{KG}}$ of the Kettani-Gubner method (column 3 of Table 2) should be taken as the most reliable value of the present analysis. The corresponding memory time constant as obtained from Table 2 (column 2) for $H_{\mathrm{KG}}=0.6-0.7$ is $\tau_{\mathrm{M}}=2000-10,000 T_{\mathrm{S}} \approx 1-3 \mathrm{~min}$.

Gao et al. (2006a, 2007) provide a critical analysis of the methods used in the present study which are related to the fluctuation analysis (FA) method of a random walk process derived from the fractional Gaussian noise process. FA, in turn, is discussed as a special case within the multifractal formalism which shows that $H$-values can be trusted if they do not take values near $H=0$ and $H=1$, a condition which is fulfilled in the analysis of the present study. The same conclusion was drawn already by Gao et al. (2006a) for their analysis of the experimental results.

The general agreement of the simulations with the experimental results in Gao et al. (2006a) $\left(H_{\mathrm{var}}=0.6-0.84\right.$, obtained for different subjects with binocular rivalry and Necker cube perspective reversal experiments) supports the fractal character of the simulated reversal time series. This finding again fits into the proposed picture of underlying 
nonlinear brain dynamics as proposed by Gao et al. (2006a) and as derived from analysis and theoretical modeling of EEG time series (e.g., Lutzenberger et al. 1995; Dafilis et al. 2001; Burke and de Paor 2004).

The prediction of the high frequency $(>5 \mathrm{~Hz})$ limit cycle and chaotic oscillations of the perception state variable of Eq. 1 (Figs. 4, 5, 6, and 7; Appendix A2 and A3; see also Ikeda and Matsumoto 1987; Derstine et al. 1987) due to the nonlinear feedback with delay $T=40 \mathrm{~ms}$, superimposed on the low frequency self-oscillations between the metastable stationary perception states $(\mathrm{P} 1 \Leftrightarrow \mathrm{P} 2)$ is consistent with recent research of these and other authors in the analysis and modeling of EEG time series. It supports the relevance of the chaotic and fractal character of brain dynamics, with additional stochastic noise components. It contradicts, however, the assumption of a purely stochastic (e.g., Poisson) process (e.g., De Marco et al. 1977; Merk and Schnakenberg 2002) as origin of the dwell time statistics.

In contrast to these authors, with the present dynamical systems approach the order of magnitude of the reversal rate (mean reversal rate $1 / \Delta_{\mathrm{m}}$ ) can be derived from a simple Thalamocortical self-oscillator model via analytical approximation (Eq. 6, Appendix A4). Without noise $\left(L_{\mathrm{t}}=0\right)$ purely periodic $\mathrm{P} 1-\mathrm{P} 2$ alternations between the stationary percepts are obtained via integration of the PAM equations, like in Ditzinger and Haken (1989). This means that the limit cycle and chaotic oscillations superimposed on the stationary states P1, P2 as depicted in Fig. 5, 6, and 7 is not the main origin of the dwell time statistics. The variance of the gamma-density of dwell times as reasonable fitting curve is determined by the stochastic noise power $\left(L_{\mathrm{t}} \neq 0\right)$, i.e., dissipative effects (e.g., Haken 2002), however, modified by memory effects (via $v_{\mathrm{b}}(t)$ ) which are quantified by the Hurst parameter.

It may be speculated that limit cycle and chaotic oscillations become important for understanding fixational eye movements (tremor and microsaccades) due to coupling of the Thalamocortical loops to the eye movement loop via SC and LGN in Fig. 1, which is neglected within the present model.

The advantage of parametric parsimony of the present scalar model of cognitive bistability with only one percept variable $v$ is paid for with the problem of non-symmetry between P1- and P2-dynamics, as mentioned already in Sect. 2.1 and depicted, e.g., in the phase space plots of Fig. 5a and $b$. This may be understood by inspection of the hysteresis curve in Fig. 3. Both the first-order S-shaped hysteresis curve and the pitchfork period doubling bifurcations show somewhat different behavior for the P1 (lower) and P2 (upper) $v$-state, giving rise to differences in limit cycle and chaotic oscillation amplitudes and mean perceptual duration times. Although, this certainly influences the exact numerical values of the statistical parameters of the numerical simulations (e.g., different mean and variance of P1 vs. P2 gamma-den- sities of perceptual duration times; Fürstenau 2004), it does not change the general conclusions.

The asymmetry may be eliminated at the cost of doubling the model complexity (doubling of number of model parameters) by means of attributing a set of PAM equations $\{(1)(2)(3)\}_{\mathrm{i}}$ to each percept $v_{i}, \quad i=1,2$, and adding an inhibitory coupling phase term $c_{\mathrm{ij}} v_{\mathrm{j}}$ to the cosargument, with typically $c_{i j} \approx 0.1$, yielding a vector model $\left\{v_{1}(t), v_{2}(t) ; G_{1}(t), G_{2}(t) ; v_{b 1}(t), v_{b 2}(t)\right\}$. An example simulation is presented in Fürstenau (2009). This is comparable to Ditzinger and Haken (1989) and similar models such as proposed recently by Wilson (1999), Hock et al. (2003), and Noest et al. (2007). With the vector model the perception state $\mathrm{P} 1\left(v^{*} \approx 1\right)$ of the present reduced model corresponds to the perception ground state $\mathrm{P} 0$ of each percept Pi (= upper level of the hysteresis curves $v_{i}^{*}\left(G_{i}\right)$, like that one of Fig. 3) which may be characterized by its own set of parameters $\left(\mu, \tau, \gamma, \tau_{\mathrm{G}}, \tau_{\mathrm{M}}, \tau_{\mathrm{L}}\right)_{i}$.

Similar to Haken's synergetic order and control parameter equations some of the other authors use two pairs of coupled perception state and adaptation equations. Noest et al. (2007), like Fürstenau (2003, 2006), emphasize the difference between the quasiperiodic perception state switching under continuous stimulus presentation and the stimulus onset dynamics (in the present model $\mu<\mu_{\mathrm{n}}=0.18 \Rightarrow$ $\mu>0.18$ ) giving rise to a node bifurcation which leads to a noise-induced biased (through $v_{\mathrm{b}}$ ) selection (choice) between the alternative percepts.

With suitable coupling between $v_{1}, v_{2}$ and zero noise, $v_{1}$ and $v_{2}$ are in full antiphase (e.g., Ditzinger and Haken 1989; Wilson 1999). Due to this redundancy, important properties of the system dynamics may be obtained with the present simplified scalar PAM-model with coupling between a single perception state order parameter $v$, an adaptive feedback gain (attention) control parameter $G$, and an adaptive bias (memory) parameter $v_{\mathrm{b}}$. The preliminary results obtained with a two-component vector model $\left\{v_{1}, G_{1}, v_{b 1} ; v_{2}, G_{2}, v_{b 2}\right\}$ show no significant deviations from the present ones with the scalar model, and they will be published separately. An example simulation of the vector model is presented in Fürstenau (2009).

An interesting aspect of the simulation results in Table 2 concerns the comparison with experimentally observed differences between binocular rivalry and ambiguous figure reversal. Whereas binocular rivalry appears to involve a more automatic, stimulus driven form of competition (Deco and Marti 2007) and exhibits no chaotic contribution in the reversal time statistics (Richards et al. 1994) (Lehky 1995), alternation rates of ambiguous figure reversal, on the other hand, show strong response to selective attention, i.e., can be voluntarily controlled by observers (Meng and Tong 2004). Moreover, by means of the correlation dimension $D_{2}$ (as another indicator of self-similarity and chaotic dynamics) 
from experimental reversal time series of ambiguous figures, Richards et al. (1994) determined a significant deterministic contribution which was not the case for rivalry. In terms of the present model, binocular rivalry corresponds to a large bias time constant $\tau_{\mathrm{M}}$ (last row of Table 2 ) with vanishing long range correlations $(H \approx 0.5)$. Within the present model, the reason for long range correlations are attributed to memory effects and they vanish if the memory time constant exceeds ca. $15 \mathrm{~min}\left(\tau_{\mathrm{M}}=50000 T_{\mathrm{S}}\right)$, i.e., if memory changes are slow.

\section{Conclusion and outlook}

Based on a previously introduced behavioral recursive nonlinear dynamics model of cognitive bistability (e.g., Fürstenau 2003, 2004, 2006, 2007) long range correlations within time series of percept changes are predicted due to perception-attention-memory (PAM) coupling. The present model represents a kind of minimum architecture and can be mapped to simplified reentrant thalamocortical circuits within the association cortex including attentional feedback modulation of the ventral ("what") stream (e.g., Itti and Koch 2001). The dynamics of the phase oscillator perception circuit is determined by reentrant adaptive gain with delay $T \approx 40 \mathrm{~ms}$ (Lamme 2003) which gives rise to selfoscillations of the order of 20 percept reversals per minute due to attention fatigue, in addition to the superimposed small delay-induced chaotic and limit cycle oscillations of the metastable perception states which for our specific perception state equation (1) was investigated in detail by Ikeda and Matsumoto (1987). The numerical analysis is confirmed by a linearized analytical approximation.

It was verified already in previous study (Fürstenau 2003, 2004) that like with the synergetic model of Ditzinger and Haken (1989), simulated perceptual reversal time statistics can be fitted by $\Gamma$-distributions with mean and variance corresponding to the experimentally observed range of some seconds (Orbach et al. 1963) (Borsellino et al. 1972; Nakatani and van Leeuwen 2005; Zhou et al. 2004). The analysis of the computer experiments shows that the variance is determined by the noise power spectral density of the attention parameter whereas the mean dwell time is determined mainly by the self-oscillator characteristics with fatigue time constant $\gamma$, damping time $\tau$ and reentrant delay $T$, however modified by the stochastic noise. That is why the present model underlines the importance of the Fluctuation-Dissipation theorem (which plays a fundamental role in physics) also for cognitive processes (e.g., Haken 2002). The estimates of the shape parameter $\alpha>1$ indicate the underlying stochastic process to be smoothed (i.e., less random) as compared to the random Poisson process characterized by maximum information entropy (Dodson and Scharcanski 2003)
The present theoretical results on long range correlations (memory effects) which reproduce the experiments of Gao et al. (2006a) indicate the necessity to extend and modify the proposed picture of a cognitive of stochastic clock with percept switching after $\alpha$ discrete cognitive processing steps (clock pulses) if integer $\alpha$ are assumed (e.g., Murata et al. 2003). For simulating memory effects, the dynamics of the attention control parameter (=feedback gain) is biased by an adaptive preference parameter which in turn is coupled to the perception state. Reversal time series exhibit long range correlations characterized by the Hurst (second-order selfsimilarity) parameter with $H>0.5$ in agreement with experimental results of Gao et al. (2006a).

Consistent $H$-values of the simulated percept reversal time series significantly exceeding $H=0.5$ (= purely stochastic process; random walk) have been derived by three different methods. The three methods are critically reviewed with regard to multifractal analysis methods analyzed by Gao et al. (2006b, 2007). Several more advanced methods are proposed by these authors for evaluating multifractal time series. This, however, was not within the scope of the present analysis, and in agreement with Gao et al. (2006a), it appears not necessary because the conditions of Gao et al. (2006b) for valid $H$ estimates are fulfilled: $H$-values are not near $H=0$ and $H=1$. Within the present model, these long range correlations decrease with increasing memory time constant $\tau_{\mathrm{M}}$, with $\tau_{\mathrm{M}}=60-200$ s yielding $H \approx 0.7-0.6$ as the most probable value (Kettani-Gubner method, column 3 of Table 2). This appears to provide a sound theoretical explanation of the experimental findings of Gao et al. (2006a) and confirms their conclusion that nonlinear brain dynamics can be assumed as origin of the long range correlations. It also agrees with their statement that a value around $H=0.7$ makes most sense because it is ubiquitous in nature.

The experimentally observed differences between binocular rivalry and ambiguous figure reversal with regard to their chaotic or fractal character (Richards et al. 1994; Meng and Tong 2004) can be attributed to different attention bias (memory) time constants.

The present nonlinear dynamics model furthermore supports the early proposal of Poston and Stewart (1978) and of Wilson (1999) of a deterministic catastrophe topology as the formal basis of the perception reversal dynamics. The inclusion of delayed feedback immediately leads to limit cycle and chaotic contributions to the spectral characteristics of metastable perception states which supports experimental and theoretical results of other authors on the chaotic character of mesoscopic brain states (e.g., Lutzenberger et al. 1995).

The present reduced model with only one percept variable may easily be extended into a vector model with separate sets of PAM-equations for each percept which allows for multistable perceptual switching between three and more stationary states. 
Ongoing work (Fürstenau 2009) is focused on the simulation of periodic (i.e., interrupted) stimulus $(\mu(t))$, comparable to Noest et al. (2007), for reproducing, e.g., the experimental results of Orbach et al. (1963). This is an experimental condition which with the present model is easily reproduced by periodic switching of the $\mu$-parameter between low (stimulus-off) and high values (stimulus-on; see Figs. 6, 7: stimulus-on at $t=500 T_{\mathrm{S}}$ ). Initial simulation results under periodic stimulus switching with variation of stimulus-off time are presented in (Fürstenau 2009) where also an example solution of the full vector model (see above) can be found. The presented minimum version of the recursive interference model does not allow for modeling multistable transitions between more than two perception states as experimentally investigated, e.g., by Ito et al. (2003). Modeling of multistability requires the nonlinear vector model with perception state vectors $\left[v_{1}, v_{2}, \ldots, v_{\mathrm{n}}\right]$ and corresponding sets of PAM-equations.

It would be of interest to compare predictions of related dynamical modeling approaches (Ditzinger and Haken 1989; Noest et al. 2007; Hock et al. 2003) with the present ones with regard to the experimentally observed long range correlations. An interesting experimental task for testing the cognitive self-oscillator hypothesis and for quantifying the relative importance of deterministic versus stochastic dynamics would be the isolation of significant peaks around $0.1-$ $0.5 \mathrm{~Hz}$ in the frequency spectrum of the dwell-time time series.

Acknowledgments I am indebted to Monika Mittendorf for help with the computer experiments and data evaluation, to H. Nakatani of Riken Brain Science Institute for information on recent experimental results, and, in particular, to J.B. Gao and K.D. White of University of Florida for providing an early preprint of their study. J. B. G. is now with PMB Intelligence LLC. Significant improvements of the original version of the manuscript are due to valuable comments and questions of the anonymous reviewers.

Open Access This article is distributed under the terms of the Creative Commons Attribution Noncommercial License which permits any noncommercial use, distribution, and reproduction in any medium, provided the original author(s) and source are credited.

\section{Appendix}

\section{A1 Perception state equation}

Time-averaged superimposition of two phase shifted fields oscillating at frequency $v=\omega / 2 \pi$ with the (complex) amplitudes: $a\left(\Phi_{1}\right)=a_{0} \exp \left\{j\left(\omega t+\Phi_{1}\right)\right\}, b\left(\Phi_{2}\right)=$ $b_{0} \exp \left\{j\left(\omega t+\Phi_{2}\right)\right\}$, yields intensity $J$ via multiplication of the sum amplitude with its complex conjugate

$$
\begin{aligned}
J & =|a+b|^{2} \\
& =J_{0}[1+\mu \cos (\Delta \Phi)]
\end{aligned}
$$

with phase difference $\Delta \Phi=\Phi_{1}-\Phi_{2}=\pi\left(v_{t}+v_{\mathrm{B}}\right)$, constant phase offset $\pi v_{\mathrm{B}}$ and contrast parameter $\mu=2 a_{0} b_{0} /\left(a_{0}^{2}+\right.$ $\left.b_{0}^{2}\right) \exp (k \Delta \Phi \Delta \omega / \omega)$, with constant $k \approx 0.25$ and relative spectral width at half maximum $\Delta \omega / \omega$. Here, a Gaussian spectral distribution is assumed (Born and Wolf 1975). For small spectral width $\Delta \omega$, the exponential $\approx 1$ and the contrast is determined by the normalized product of amplitudes. For $a_{0}=b_{0} \mu$ is given by the exponential as contrast function. For the present reduced model with a single perception state equation for percepts $\mathrm{P} 1, \mathrm{P} 2, \mu$ is interpreted as difference-of-meaning of both percepts.

Phase feedback with delay $T$ is obtained via $v(t)=$ $K J(t)=J / J_{\pi}$, with modulation constant $K=\kappa g=$ $1 / J_{\pi}=$ modulation strength for $\pi$ phase shift. Phase difference at time $t$ is modulated by superimposed fields at time $t-T$ with delay $T=40 \mathrm{~ms}$ of the amplified feedback signal. In turn, $J(t)=J_{\pi} v(t)$, so that we get

$v(t+T)=f(v(t))=G\left[1+\mu \cos \left(\pi\left(v(t)+v_{\mathrm{B}}\right)\right)\right]$

with attention parameter $G=\kappa g J_{0}$ proportional to feedback gain and stimulus strength $J_{0}$. For a more realistic perception dynamics, a differential term $\tau \dot{v}(t+T)$ with damping time constant $\tau$ (typically of the order of $T$ ) is added to the left-hand side of Eq. A1.2, yielding the well-known differential-delay equation of bistable optical systems (Ikeda and Matsumoto 1987; Busenberg and Martinelli 1991). For simplifying the solution of the PAM Eqs. 1-3, we assume $\mu$ to be constant (corresponding to small spectral width $\Delta \omega / \omega$, i.e., high degree of coherency).

The time-independent stationary state of Eq. 1 and A1.2 is defined by $\mathrm{d} v / \mathrm{d} t=0$ and $v(t+T)=v(t)=v^{*}$ yielding the first-order stationary solution

$v^{*}=G\left[1+\mu \cos \left\{\pi\left(v^{*}+v_{\mathrm{B}}\right)\right\}\right]$.

The corresponding hysteresis curve of Fig. 3 may be easily plotted by means of the explicit form $G\left(v^{*}\right)$. Higher order stationary solutions exhibiting so-called period doubling are defined by $v\left(t+2^{\mathrm{i}} T\right)=v(t)$ and $v^{\left(2 \mathrm{i}_{*}\right)}=f^{\left(2 \mathrm{i}_{*}\right)}\left(v^{*}\right)$. For example, the second order solution with period $2 T$ is given by

$$
\begin{aligned}
v^{* *} & =f\left(f\left(v^{*}\right)\right) \\
& =g\left[1+\mu \cos \left\{\pi\left(G\left[1+\mu \cos \left\{\pi\left(v^{*}+v_{\mathrm{B}}\right)\right\}+v_{\mathrm{B}}\right]\right)\right\}\right]
\end{aligned}
$$

which starts the period doubling route to chaotic behavior of the present cos-mapping function (similar to the circle (sine) map; Anishchenko et al. 2003). The period doubling behavior was originally described by Feigenbaum (1979) for the quadratic map (see Appendix A2 and Fig. 3b) and proven to represent a universal behavior for a large class of nonlinear maps. 
A2 Transition into chaos: Lyapunov exponent

The ranges of the attention control parameter $\mathrm{G}$ for the chaotic regimes in the bifurcation graph of Fig. 3 can be determined by numerical evaluation of the Lyapunov coefficient (Schuster and Just 2005) which for Eq. 1 is given by

$\lambda\left(v_{0}\right)=\lim _{N \rightarrow \infty} \sum_{\mathrm{i}=0}^{N-1} \ln \left(\left|f^{\prime}\left(v_{\mathrm{i}}\right)\right|\right)$

with $N=$ number of iteration steps and $f^{\prime}\left(v_{\mathrm{i}}\right)=$ derivative of the RHS. of the nonlinear mapping function. We derive $f^{\prime}(v)$ for the discrete map where the continuous time derivative $\mathrm{d} v / \mathrm{d} t$ is approximated by $\left(v_{\mathrm{t}+\mathrm{T}}-v_{\mathrm{t}}\right) / T$ yielding

$v_{\mathrm{t}+\mathrm{T}}=\frac{\tau / T}{1+\tau / T} v_{\mathrm{t}}+\frac{G}{1+\tau / T}\left(1+\mu \cos \left[\pi\left(v_{\mathrm{t}}+v_{\mathrm{B}}\right)\right]\right)$.

The derivative then is obtained as

$f^{\prime}(v)=-\frac{\pi G \mu}{1+\tau / T} \sin \left(\pi\left(v-v_{\mathrm{B}}\right)\right)+\frac{\tau / T}{1+\tau / T}$

$\lambda$ measures the exponential separation of neighboring initial states $v_{0}$ during the iteration process under the action of the mapping. It corresponds to the average loss of information per iteration. Figure 4 shows $\lambda$ for $N=1,000$ iterates with $\tau=0$ as plotted versus $G$. Coming from negative values, $\lambda$ touches the $\lambda=0$ axis at bifurcation points $G_{n}$ (period doubling) with decreasing distance $G_{n}-G_{n-1}$ until it crosses $\lambda=0$ at the boundary to chaotic regimes, characterized by positive $\lambda$.

The first four $G_{\mathrm{n}}$-values as derived from the data of the $\lambda(\mathrm{G})$ graph in Fig. 4 for the first (P1) and second (P2) level of the stationary solutions in Fig. 3 are: $G_{1,2,3,4}=$ $(0.535,0.947,1.058,1.085)$ and $(1.368,1.448,1.467$, $1.4715)$, respectively. Within the regions around $1.1<$ $G<1.2$ and $1.48<G<1.8$, on perception levels $\mathrm{P} 1$ and $\mathrm{P} 2$, respectively, of Fig. 3b clearly chaotic behavior is observed $(\lambda>0)$. According to Feigenbaum's Universality Theory (Feigenbaum 1979), the bifurcation points $r_{\mathrm{n}}$ of the control parameter $r$ of a wide class of nonlinear maps $y_{\mathrm{k}+1}=f_{\mathrm{r}}\left(y_{\mathrm{k}}\right)$ including the quadratic one converge at the chaotic boundary governed by the universal constant $\delta_{\infty}=4.6692=\lim _{\mathrm{n} \rightarrow \infty}\left(r_{n}-r_{n-1}\right) /\left(r_{n+1}-r_{n}\right)$. The $G_{\mathrm{n}}$ values $(n=2,3,4)$ of the present circle (cos) map (Fürstenau 2004) yield the approximations $\hat{\delta}_{\infty}=4.00$ and 4.22 for the P1 and P2 levels respectively. The positive slope intersections of $\lambda(\mathrm{G})$ with the $\lambda=0$ line as observed near $G=1.1$ and $G=1.5$ in Fig. 4 for the P1 and P2 levels of Fig. 3b, respectively, agree with the numerical estimates $\hat{G}_{\infty}^{(1)} \approx 1.091$ and $\hat{G}_{\infty}^{(2)} \approx 1.473$ for the chaotic boundaries obtained with Feigenbaum's more exact number (by replacing $G_{\mathrm{n}+1}=G_{5}:=G_{\infty}$ ). It shows that within certain param- eter ranges $(G, \mu, \tau, T)$ the $\mathrm{P} 1-, \mathrm{P} 2$-self-oscillations exhibit transitions between limit cycle and chaotic dynamical states.

\section{A3 Small oscillations: linear stability analysis}

The stability of the perception state $v(t)$ versus feedback gain (attention parameter $G$ ) as dependent on low pass filter time constant $\tau$ and feedback delay $T$ is analysed via linear stability analysis of Eq. 1.

For linearization as usual small deviations $x=v-v^{*}$ from the stationary values $v^{*}$ are investigated. Introducing the ansatz $x=\exp \{(\alpha+i \beta) t\}$ into (1) with damping constant $\alpha$ and frequency $\beta$ two equations are obtained from the real and imaginary part

$$
\begin{aligned}
& \alpha+\frac{1}{\tau}+\frac{\pi G \mu}{\tau} \sin \left\{\pi\left(v^{*}+v_{\mathrm{B}}\right)\right\} \exp \{-\alpha T\} \cos \{\beta T\}=0 \\
& \beta-\frac{\pi G \mu}{\tau} \sin \left\{\pi\left(v^{*}+v_{\mathrm{B}}\right)\right\} \exp \{-\alpha T\} \sin \{\beta T\}=0 .
\end{aligned}
$$

Onset of oscillations become possible with damping constant $\alpha>0$, with boundary of instable areas defined by $\alpha=0$, yielding eigenfrequencies through combination of (A3.1) and (A3.2):

$\beta \tau=-\tan (\beta T)$.

In the simulations, we assume low damping $\tau<T$. Because $\beta<1 / \tau$, we obtain with an analytical approximation for the eigenfrequencies with $\beta \tau<\beta T<<1$ by $f=\beta / 2 \pi \approx i f_{1} /(1+\tau / T) \approx i f_{1}=i / 2 T=$ $i 12.5 \mathrm{~Hz}, i=1,2,3, \ldots$.

The explicit expression for the boundary of instability $(\alpha=0)$ is obtained through combination of the squared Eqs. A3.1 and A3.2 for eliminating the trigonometric functions of frequency $\beta$, and Taylor expansion of $\exp \{-\alpha T\}$ up to the linear term:

$v^{*}=\frac{1}{\pi} \arcsin \left\{\frac{\sqrt{(\beta \tau)^{2}+1-\tau / T}}{2 \pi \mu G}\right\}-v_{\mathrm{B}}$.

For small $\tau(<<T)$, the areas of instability extend from the first bifurcation point in Fig. $3 \mathrm{~b}$ to the right (increasing feedback gain parameter $G$ ), i.e., as expected instable areas coincide with ranges of limit cycle and chaotic oscillations (Fürstenau 2003).

\section{A4 Perception switching: self-oscillator equation}

The fatigue-induced self-oscillations of the reentrant perception-attention loop as described by the coupled stochastic differential-delay Eqs. 1 and 2 can be solved in its original 
form only numerically. An estimate of the average perceptual dwell time and self-oscillator frequency, respectively, may be obtained by solving the linearized system of nonlinear perception state (order parameter) equation coupled with the adaptive gain (control parameter) equation without noise. Introduction of attention parameter Eq. 2 into the derivative of (1) (second-order nonlinear differential equation) yields

$$
\begin{aligned}
\tau \ddot{v}_{t+T} & +\dot{v}_{\mathrm{t}+\mathrm{T}}+\pi \mu G_{\mathrm{t}} \dot{v}_{\mathrm{t}} \sin \left(\pi v_{\mathrm{t}}\right) \\
& =f\left(G_{\mathrm{t}}, v_{\mathrm{t}}\right)\left[1+\mu \cos \left(\pi v_{\mathrm{t}}\right)\right]
\end{aligned}
$$

with the fatigue function $f(G, v)$ corresponding to the RHS. of Eq. 2 without noise.

In order to obtain an analytical estimate of the self-oscillation frequency of this nonlinear delay-differential equation, the delay in the differentials is eliminated according to MacDonald (1989) for small $T$ by means of first order Taylor expansion about $T=0$ which shifts the delay effect from the damping term to the acceleration and from the acceleration to a third order term: $T \ddot{v}_{\mathrm{t}}$ and $\tau T \ddot{v}_{\mathrm{t}}$. In what follows the third order term (variation of the acceleration) will be neglected. I furthermore treat the case of small hysteresis $\mu=0.2<<1$ near the node bifurcation threshold $\mu=0.18$ which shifts the stationary solution of $v(t)$ to $v^{*} \approx 1.5$, which in turn lies near the selected perception bias $v_{\mathrm{b}}=1.5$ for the symmetric case, with $G^{*}\left(v^{*}\right) \approx 1.5 \approx G_{\text {mean }}(\mu=0.2)=1.46$.

Because for the numerical solutions in Sect. 3, the fatigue time constant $\gamma=60 T_{\mathrm{S}}<<$ recovery time constant $\tau_{\mathrm{G}}=$ $500 T_{\mathrm{S}}$ (simulation time step $T_{\mathrm{S}}=T / 2=20 \mathrm{~ms}$ ) the recovery term $\left(G_{\text {mean }}-G\right) / \tau_{\mathrm{G}}$ in the fatigue function may be neglected and Eq. A4.1 approximated by the linearized oscillator equation (e.g., Magnus 1961) using $v=v^{*}+w$, with $w=$ small deviation from $v^{*}$ :

$$
(\tau+T) \ddot{w}+\left(1-\pi \mu G^{*}\right) \dot{w} \approx-w / \gamma
$$

with eigenfrequency

$$
\omega_{0}=1 / \sqrt{\gamma(\tau+\mathrm{T})}=3.73 \mathrm{rad} \mathrm{s}^{-1}
$$

or $f_{0}=0.59 \mathrm{~Hz}=36 \mathrm{~min}^{-1}$ and $T_{0}=1.7 \mathrm{~s}$, respectively. The influence of the damping term (coefficient of $\mathrm{d} w / \mathrm{d} t$ ) can be derived after transformation of the timescale into Eigentime $\vartheta=\omega_{0} t$ (Magnus 1961):

$$
f_{\mathrm{D}}=f_{0} \sqrt{1-D^{2}}
$$

with normalized damping

$$
D=\sqrt{\frac{\left(1-\pi \mu G^{*}\right)}{2 \omega_{0}(\tau+T)}}
$$

yielding the reversal rate $f_{\mathrm{D}}=0.55 \mathrm{~Hz}=33 \mathrm{~min}^{-1}$.

\section{References}

Anishchenko VS, Astakhov VV, Neiman AB, Vadivasova TE, Schimanski-Geier L (2003) Nonlinear dynamics of chaotic and stochastic systems. Springer, Berlin

Arnold DA, Grove PM, Wallis TSA (2007) Staying focused: a functional account of perceptual suppression during binocular rivalry. $\mathrm{J}$ Vis $7: 1-8$

Atmanspacher H, Filk T, Römer H (2004) Quantum Zeno features of bistable perception. Biol Cybern 90:33-40

Beran J (1992) Statistical methods for data with long-range dependence. Stat Sci 7:404-427

Blake R, Logothetis NK (2002) Visual competition. Nat Rev Neurosci $3: 1-11$

Born M, Wolf E (1975) Principles of optics, 5th edn. Pergamon Press, Oxford

Borsellino A, de Marco A, Allazetta A, Rinesi S, Bartolini B (1972) Reversal time distribution in the perception of visual ambiguous stimuli. Kybernetik 10:139-144

Braskamp JE, van Ee R, Noest AJ, Jacobs RH, van den Berg AV (2006) The time course of binocular rivalry reveals a fundamental role of noise. J. Vis 6:1244-1256

Burke DP, de Paor AM (2004) A stochastic limit cycle oscillator model of the EEG. Biol Cybern 91:221-230

Busenberg S, Martinelli M (eds) (1991) Delay differential equations and dynamical systems. Lecture Notes in Mathematics, vol 1475. Springer, New York

Dafilis MP, Liley DTJ, Cadusch PJ (2001) Robust chaos in a model of the electroencephalogram: Implications for brain dynamics. Chaos 11:474-478

Deco G, Marti D (2007) Deterministic analysis of stochastic bifurcations in multi-stable neurodynamical systems. Biol Cybern 96:487-496

deGuzman GC, Kelso JAS (1991) Multifrequency behavioral patterns and the phase attractive circle map. Biol Cybern 64:485-495

De Marco A, Penengo P, Trabucco A, Borsellino A, Carlini F, Riani M, Tuccio MT (1977) Stochastic models and fluctuations in reversal time of ambiguous figures. Perception 6:645-656

Derstine MW, Jewell JL, Gibbs HM, Hopf FA, Rushford MC, Sanders LD, Tai K (1987) Experimental verification of regenerative pulastions and chaos. In: Arecchi FT, Harrison RG (eds) Instabilities and chaos in quantum optics. Springer Series in Synergetics, vol 34. Springer, Berlin, pp 175-198

Ditzinger T, Haken H (1989) Oscillations in the perception of ambiguous patterns. Biol Cybern 61:279-287

Ditzinger T, Haken H (1995) A synergetic model of multistability in perception. In: Kruse P, Stadler M (eds) Ambiguity in mind and nature. Springer, Berlin, pp 255-273

Dodson CTJ, Scharcanski J (2003) Information geometric similarity measurement for near-random stochastic processes. IEEE Trans Syst Man Cybern A 33:435-440

Edelman G (2004) Wider than the sky. Penguin Books, New York, pp $87-96$

Engel AK, Fries P, König P, Brecht M, Singer W (1999) Temporal binding, binocular rivalry, and consciousness. Conscious Cogn 8:128151

Engel AK, Fries P, Singer W (2001) Dynamic predictions: oscillations and synchrony in top-down processing. Nat Rev Neurosci 2:704 718

Feigenbaum MJ (1979) The universal metric properties of nonlinear transformations. J. Stat Phys 21:669-706

Frank TD, Michelbrink M, Beckmann H, Schöllhorn WI (2008) A quantitative dynamical systems approach to differential learning: self-organization principle and orderparameter equations. Biol Cybern 98:19-31 
Freeman WJ (2000) Neurodynamics: an exploration in mesoscopic brain dynamics. Springer, Berlin

Fürstenau N (2003) Nonlinear dynamics model of cognitive multistability and binocular rivalry. Proceedings IEEE 2003 International Conference on Systems, Man and Cybernetics, IEEE cat. no. $03 \mathrm{CH} 37483 \mathrm{C}$, pp 1081-1088

Fürstenau N (2004) A chaotic attractor model of cognitive multistability. Proceedings IEEE 2004 International Conference on Systems, Man and Cybernetics, IEEE cat. no. 04CH37583C, pp 853-859

Fürstenau N (2006) Modelling and simulation of spontaneous perception switching with ambiguous visual stimuli in augmented vision systems. Lecture Notes in Artificial Intelligence, vol 4021. Springer, Berlin, pp 20-31

Fürstenau N (2007) A computational model of bistable perceptionattention dynamics with long range correlations. In: Hertzberg J, Beetz M, Englert R (eds) KI2007, Lecture Notes in Artificial Intelligence LNAI 4667. Springer, Berlin, pp 251-263

Fürstenau N (2009) Computational nonlinear dynamics model of percept switching with ambiguous stimuli. In: Duffy VG (ed) HCII2009, Lecture Notes in Computer Science, LNCS 5620. Springer, Berlin, pp 227-236

Gao JB, Merk I, Tung WW, Billok V, White KD, Harris JG, Roychowdhury VP (2006a) Inertia and memory in visual perception. Cogn. Process 7:105-112

Gao J, Hu J, Tung WW, Yinhe C, Sarshar N, Roychowdhury VP (2006b) Assessment of long-range correlation in time series: how to avoid pitfalls. Phys Rev E 73:016117-1-016117-10

Gao J, Hu J, Tung WW, Cao YH (2006c) Distinguishing chaos from noise by scale dependent Lyapunov exponent. Phys Rev E 74:066204-1-066204-9

Gao J, Cao Y, Tung W-W, Jing H (2007) Multiscale analysis of complex time series. Wiley-Interscience, Hoboken, NJ

Haken H (1978) Synergetics, 2nd edn. Springer, Berlin

Haken H (2002) Brain dynamics. Springer, Berlin

Haken H, Kelso JAS, Bunz H (1985) A theoretical model for phase transitions in human movement. Biol Cybern 53:247-257

Hamker FH (2004) A dynamic model of how feature cues guide spatial attention. Vis Res 44:501-521

Hillyard SA, Vogel EK, Luck SJ (1999) Sensory gain control (amplification) as a mechanism of selective attention: electrophysiological and neuroimaging evidence. Philos Trans R Soc Lond B 353(1373):1257-1270

Hock HS, Kelso JAS, Schöner G (1993) Bistability and hysteresis in the organisation of apparent motion patterns. J Exp Psychol 19: 63-80

Hock HS, Schöner G, Voss A (1997) The influence of adaptation and stochastic fluctuations on spontaneous perceptual changes for bistable stimuli. Percep Psychophys 59:509-522

Hock HS, Schöner G, Giese M (2003) The dynamical foundations of motion pattern formation: stability, selective adaptation, and perceptual continuity. Percep Psychophys 65:429-457

Ikeda K, Matsumoto K (1987) High dimensional chaotic behavior in systems with time delayed feedback. Physica 29D:223-235

Ito J, Nikolaev AR, Luman M, Aukes MF, Nakatani C, van Leeuwen C (2003) Perceptual switching, eye movements, and the bus paradox. Perception 32:681-698

Itti L, Koch C (2001) Computational modelling of visual attention. Nat Rev Neurosci 2:194-203

Jirsa V, Haken H (1996) Field theory of electromagnetic brain activity. Phys Rev Lett 77(5):960-963

Jirsa V, Haken H (1997) A derivation of a macroscopic field theory of the brain from quasi microscopic neural dynamics. Physica D99:503-526

Kelso JAS (1995) Dynamic patterns: the self-organization of brain and behavior. The MIT Press, Cambridge, London
Kelso JAS, Bressler SL, Buchanan S, DeGuzman GC, Ding M, Fuchs A, Holroyd T (1992) A phase transition in human brain and behavior. Phys Lett A 169:134-144

Kelso JAS, Case P, Holroyd T, Horvath E, Raczaszek J, Tuller B, Ding M (1995) Multistability and metastability in perceptual and brain dynamics. In: Kruse P, Stadler M (eds) Ambiguity in mind and nature. Springer, Berlin, pp 255-273

Kettani H, Gubner JA (2006) A novel approach to the estimation of the long-range dependence parameter. IEEE Trans Circ Syst II $53: 463-467$

Lamme VAF (2003) Why visual attention and awareness are different. Trends Cogn Sci 7:12-18

Levelt WJM (1967) Note on the distribution of dominance times in binocular rivalry. Br J Psychol 58:143-145

Lehky SR (1995) Binocular rivalry is not chaotic. Proc R Soc Lond B 259:71-76

Loxley PN, Robinson PA (2009) Soliton model of competitive neural dynamics during binocular rivalry. Phys Rev Lett 102:258701-1258701-4

Lutzenberger W, Preissl H, Pulvermüller F (1995) Fractal dimension of electroencephalographic time series and underlying brain processes. Biol Cybern 73:477-482

MacDonald N (1989) Biological delay systems: linear stability analysis. Cambridge University Press, Cambridge

Magnus K (1961) Schwingungen. Teubner, Stuttgart

Mandelbrot BB (1991) The fractal geometry of nature (German translation). Birkhäuser, Basel, pp 265-270

Meng M, Tong F (2004) Can attention selectively bias bistable perception? Differences between binocular rivalry and ambiguous figures. J. Vis 4:539-551

Merk ILK, Schnakenberg J (2002) A stochastic model of multistable perception. Biol Cybern 86:111-116

Murata T, Matsui N, Miyauchi S, Kakita Y, Yanagidu T (2003) Discrete stochastic process underlying perceptual rivalry. Neuroreport 14:1347-1352

Nakatani H, van Leeuwen C (2005) Individual Differences in Perceptual Switching rates: the role of occipital alpha and frontal theta band activity. Biol Cybern 93:343-354

Nakatani H, van Leeuwen C (2006) Transient synchrony of distant brain areas and perceptual switching in ambiguous figures. Biol Cybern 94:445-457

Natsuki N, Nishimura H, Matsui N (2000) A neural chaos model of multistable perception. Neural Process Lett 12:267-276

Noest AJ, van Ee R, Nijs MM, van Wezel RJA (2007) Percept-choice sequences driven by interrupted ambiguous stimuli: a low-level neural model. J Vis 7:1-14

Orbach J, Ehrlich D, Heath HA (1963) Reversibility of the Necker cube: an examination of the concept of satiation of orientation. Percep Motor Skills 17:439-458

Patterson R, Winterbottom M, Pierce B, Fox R (2007) Binocular rivalry and head worn displays. Hum Fact 49:1083-1096

Pitts MA, Nerger JL, Davis TJR (2007) Electrophysiological correlates of perceptual reversals for three different types of multistable images. J Vis 7:1-14

Poston T, Stewart I (1978) Nonlinear modeling of multistable perception. Behav Sci 23:318-334

Richards W, Wilson HR, Sommer MA (1994) Chaos in percepts. Biol Cybern 70:345-349

Robinson D (ed) (1998) Neurobiology. Springer, Berlin

Robinson PA (2005) Propagator theory of brain dynamics. Phys Rev E72:011904-1-011904-14

Schuster HG, Just W (2005) Deterministic chaos, 4th edn. Wiley-VCH, Weinheim

Schuster HG, Wagner PA (1990) A model for neural oscillations in the visual cortex: 1 . Mean field theory and the derivation of the phase equations. Biol Cybern 64:77-82 
Srinavasan R, Russel DS, Edelman GM, Tononi G (1999) Increased synchronization of magnetic responses during conscious perception. J Neurosci 19:5435-5448

Tononi G, Edelman GM (1998) Consciousness and complexity. Science 282:1846-1851

von der Malsburg C (1997) The coherence definition of consciousness. In: Ho M, Miyashita Y, Rolls ET (eds) Cognition, computation, and consciousnesss. Oxford University Press, v, pp 193-204
Watts C, Fürstenau N (1989) Multistable fiber-optic Michelson Interferometer exhibiting 95 stable states. IEEE J Quantum Electron $25: 1-5$

Wilson HR (1999) Spikes, decisions, and actions. Oxford University Press, Oxford

Zhou YH, Gao JB, White KD, Merk I, Yao K (2004) Perceptual dominance time distributions in multistable visual perception. Biol Cybern 90:256-263 